\title{
Fundamental Analysis of the Perturbation Rejection Characteristics of Single-Input-Multiple-Output Systems Subject to Multiple Perturbations
}

\author{
Luis Amezquita-Brooks, ${ }^{1}$ Jesús Ulises Liceaga-Castro, ${ }^{2}$ Eduardo Liceaga-Castro, ${ }^{1}$ \\ Daniel Martinez-Vazquez, ${ }^{1}$ and Octavio Garcia-Salazar ${ }^{1}$ \\ ${ }^{1}$ Centro de Investigación e Innovación en Ingeniería Aeronáutica, Facultad de Ingeniería Mecánica y Eléctrica, \\ Universidad Autónoma de Nuevo León, San Nicolas de los Garza, NL, Mexico \\ ${ }^{2}$ Departamento de Electrónica, Universidad Autónoma Metropolitana Unidad Azcapotzalco, Mexico City, Mexico \\ Correspondence should be addressed to Luis Amezquita-Brooks; luis.amezquitabrk@uanl.edu.mx
}

Received 9 October 2016; Revised 30 March 2017; Accepted 11 April 2017; Published 19 June 2017

Academic Editor: Francisco Gordillo

Copyright (C 2017 Luis Amezquita-Brooks et al. This is an open access article distributed under the Creative Commons Attribution License, which permits unrestricted use, distribution, and reproduction in any medium, provided the original work is properly cited.

\begin{abstract}
Single-Input-Multiple-Output (SIMO) systems are found in several applications. Some of the main concerns are (1) the possibility of stabilizing all the outputs and (2) the possibility of attaining independent tracking control of all the outputs. Whereas the first issue can be easily be elucidated, the second has proven to be impossible in all but a few systems. In many cases one practical option is to use the input to drive a main output, taking care that the behavior of the remaining secondary outputs is acceptable. In this configuration, in addition to the features of the main control loop, the perturbation rejection properties of the secondary outputs become important. This article analyzes the structural properties, stability, and perturbation rejection characteristics of SIMO systems. The article presents fundamental conclusions regarding the relationship of the main control loop and the perturbation rejection characteristics of the secondary outputs. A simple and intuitive example is used to show how the theoretical findings can be used to improve the design of the main control loop through its frequency domain characteristics. The results are developed using simple frequency domain theoretical elements, making the findings relevant for both engineering applications and deriving further theoretical developments.
\end{abstract}

\section{Introduction}

Single-Input-Multiple-Output (SIMO) systems appear on many industrial and technological applications where there is the need for closed-loop control, for instance, in electric machine control [1,2], airplane control [3-5], and automotive active suspension control [6].

The difficulty of such systems is that in most cases it is not possible to attaint total control of all outputs using a single input. In particular, fully independent reference tracking control may be impossible [7]. In some cases a bandwidth separation and nested closed-loop control allow a degree of control over several output variables. This strategy has been historically used with success in aeronautical applications and other electromechanical systems [8-10].

The stability of SIMO control systems is an issue of ongoing interest. For instance, in [11, 12] the stability of state feedback SIMO systems are studied from a theoretical point of view. While the stability of any control system is of prime importance, it is well-known that for actual practical applications the stability is only a starting requirement.

Other more common strategy which uses modern control tools is the use of optimal controllers in which all the outputs are considered on the cost function [7, 13]. While these control approaches may yield good results, the design procedure for such controllers still requires the calibration of the 
weighting matrixes. In addition, this design process lacks the elements to closely relate the control system specifications with perturbation rejection for all the outputs.

Another important characteristic of many SIMO plants is that although only one control input is available, there may be numerous perturbation inputs. For example, in classic aircraft control the elevators angle is used as the main control input for altitude, pitch, and attack angles. Nonetheless, altitude, pitch, and attack angles dynamics are also subject to many external perturbations such as wind gusts, changes in load, and other atmospheric phenomena. If sufficient knowledge of the nature of the perturbations is available, the effects of these external perturbations can be modeled as unknown perturbation inputs feeding known perturbation dynamics. For instance, while the speed of the wind gust may be unknown, the dynamical effects of the wind gust for a given speed are known; thus an unknown input (the wind gust speed) is used as input to a known perturbation dynamic. The resulting model is, in a sense, Multiple Inputs MultipleOutputs (MIMO) because it has multiple inputs. However, only one of these inputs is a control variable. In this article this type of systems is referred to as SIMO systems subject to multiple perturbations.

In [14] it is shown that there is a close relationship between the sensitivity of SIMO systems outputs and the controller open-loop gain characteristics. In addition, it is known that the sensitivity characteristics are important for perturbation rejection. However, this study and the ones mentioned before do not consider additional external perturbations directly. Nevertheless, since the consideration of external perturbations is crucial for most practical applications these aspects should be investigated in order to complete a useful design procedure.

There are several control approaches that allow dealing with external perturbations. For instance, in [15], Active Disturbance Rejection Control ( $A D R C)$ is used in combination with a decoupling strategy to control several MIMO systems successfully. Although in [15] the MIMO case was studied, these concepts could be adapted to the SIMO case. On the other hand, ADRC does not consider explicit knowledge of the perturbation dynamics. In these cases ADRC could be a good alternative. However, if further knowledge of the perturbation dynamics is available it will be shown that several important conclusions can be elucidated. Moreover, the theoretical tools developed in this article do not impose any restriction on the characteristics of the controller, allowing the study of other control strategies, such as ADRC, under the scope of perturbation rejection to multiple external perturbations.

In many SIMO tracking control systems a main output is required to follow a prescribed trajectory, while the complementary or secondary outputs remain asymptotically stable. The nested control loops strategy arises with the purpose of accomplishing this condition. In most engineering applications a further analysis of the resulting dynamics of the secondary variables may be necessary. The main reason behind this is that whereas full tracking control of such variables may be not required, these variables are often required to evolve in a neighborhood of the equilibrium point. That is, the process must operate within the desired operating regime.

There are several practical examples of this phenomenon in control applications. For instance, in motor control the main output variable is the generated torque; however, the machine flux is often required to operate within a restricted range. While the flux operates within this range the motor often operates without problem and the effect of flux variations over the output may not be evident. However, if the flux is driven out of certain neighborhood of the ideal equilibrium point, the machine may bifurcate into another equilibrium point causing an undesired behavior in the other system variables $[1,2,16]$.

Another example is airplane altitude control using the elevators. In this case the main variable is the altitude. However, the angle of attack is also affected by the elevators, and it should be maintained within certain range. If the angle of attack increases to certain value the wing stalls and the overall dynamics of the altitude suffer a heavy transformation, which may result in unrecoverable loss of airplane control [3].

In many applications the system dynamics may be properly approximated by linear models if all the output variables of the SIMO system are properly regulated. This allows the use of linear control tools for the design of adequate controllers. However, as discussed before, the stabilization of the system may not be sufficient in the presence of perturbations due to the possible deviation of secondary outputs from the operating range because of external perturbations.

Considering that many SIMO systems are designed considering only the control of the main output variable then the relationship between this main control loop and the perturbation rejection characteristics of the secondary outputs becomes relevant. If the case is that increasing the performance for the main variable decreases the performance and robustness of the secondary variables then what controller for the main variable is indicated in order to meet the overall system specifications? Moreover, in the presence of input perturbations, how can the designer assess the sensitivity and rejection of such perturbations? It is especially of interest to know how a high performance control of the main variable will affect the other variables perturbation rejection capabilities. Some of these questions are partially answered in $[13,14]$. However, these articles fail to fully characterize all the loops interactions, the external perturbations, and nonsquare SIMO systems.

In this article, the SIMO problem is addressed using a multivariable control analysis and design framework known as Individual Channel Analysis and Design (ICAD) [17]. The ICAD framework allows using classical single input single output (SISO) measures of robustness and performance for multiple input multiple output (MIMO) systems. The ICAD framework allows the interpretation of complex control problems in the context of engineering systems [8, 16, 18-21]. Although ICAD was originally proposed as a decentralized control design tool, in this article ICAD is adapted for the study of the SIMO perturbation rejection problem. The main advantage of ICAD is that the results can be readily interpreted in the frequency domain. This enables intuitive 
engineering interpretations and opens the door for further frequency analysis formalism such as $H_{\infty}$.

By adapting ICAD to the nonsquare SIMO control problem, conditions for stability of SIMO control systems subject to perturbations are determined. Moreover, the stability of the complete control system sometimes may not reflect all the information from the design point of view. Therefore, the stability of the SIMO control system is divided on sets which intuitively relate to specific parts of the control system. The analysis allows showing directly the performance and perturbation rejection tradeoffs with classical frequency analysis tools such as Bode plots. This allows the designer to take better-informed design decisions relating the technical specifications and the controller. The authors hope that this may aid in bridging the theory-practice gap on such problems. It should be noted that some of the main ideas presented here formally were first explored within the realm of a practical application, in particular, during the analysis of the structural characteristics of the flux-torque subsystem of induction motors [16]. Finally, the acronyms used along the article are shown in Acronyms at the end of the paper.

\section{Problem Statement}

Consider the following input-perturbed SIMO system:

$$
\left.\begin{array}{rl}
Y(s) & =G_{u}(s) u(s)+G_{p}(s) P_{e}(s) \\
Y(s) & =\left(\begin{array}{c}
y_{1}(s) \\
y_{2}(s) \\
\vdots \\
y_{n}(s)
\end{array}\right) \\
P_{e}(s)= & \left(\begin{array}{c}
p_{e 1}(s) \\
p_{e 2}(s) \\
\vdots \\
p_{e m}(s)
\end{array}\right) \\
G_{u}(s)= & \left(\begin{array}{c}
g_{1, u}(s) \\
g_{2, u}(s) \\
\vdots \\
g_{3, u}(s)
\end{array}\right) \\
G_{p}(s)= & \left(\begin{array}{c}
g_{1, p 1}(s) \\
g_{2, p 1}(s) \\
\vdots \\
g_{n, p 1}(s)
\end{array}\right) g_{n, p 2}(s) \cdots g_{n, p m}(s)
\end{array}\right)
$$

where $Y(s)$ is the output vector, $u(s)$ is the scalar input signal, $P_{e}(s)$ is an unknown perturbation signal vector, $G_{u}(s)$ is the unperturbed nominal SIMO plant, and $G_{p}(s)$ is an additive perturbation matrix. In this case the system contains one input, $n$ outputs, and $m$ unknown perturbation signals.
The goal is to elucidate the effect of the perturbations $P_{e}(s)$ over the outputs $Y(s)$ when a particular output is controlled using the input $u(s)$. Without loss of generality, consider that the main output is $y_{1}(s)$ and the remaining outputs are secondary outputs. If the main output is controlled using a feedback controller $k(s)$ then

$$
\begin{aligned}
& u(s)=k(s) e(s) \\
& e(s)=\operatorname{ref}(s)-y_{1}(s),
\end{aligned}
$$

where $\operatorname{ref}(s)$ is the main output reference and $e(s)$ is the error. In this case it is considered that the secondary outputs must converge to the designated equilibrium which is equal to zero for (1) if the system is operated in regulation. The resulting control scheme is shown in Figure 1.

The first design task is the main closed-loop $y_{1}(s) /$ $\operatorname{ref}(s)=h(s)$ :

$$
\frac{y_{1}(s)}{\operatorname{ref}(s)}=h(s)=\frac{k(s) g_{u, 1}(s)}{1+k(s) g_{u, 1}(s)}
$$

which may be designed according to classical SISO control considerations.

Next, the effect of the perturbations over the main output variable is analyzed. Let $y_{j p}(s)=\sum_{i=1}^{m} g_{j, p i}(s) p_{e i}(s)$; that is, $y_{1 p}(s)$ contains the cumulative effect of all the perturbation signals over the main output. According to Figure 1, it is clear that $y_{1 p}(s)$ can be treated as an output perturbation acting on the main control loop. Thus, the effect of $y_{1 p}(s)$ over the main output can be modeled using the sensitivity function of the main control loop:

$$
S(s)=\frac{1}{1+k(s) g_{u, 1}(s)} .
$$

Accordingly, the effect of the perturbation vector $P_{e}(s)$ over the output $y_{1}(s)$ is given by $S(s) y_{1 p}(s)$. Then, the complete main output yields

$$
y_{1}(s)=h(s) \operatorname{ref}(s)+S(s) \sum_{i=1}^{m} g_{1, p i}(s) p_{e i}(s) .
$$

Equation (5) shows that the characteristics of the main output correspond to that of a typical perturbed SISO control system where a high bandwidth controller (that is, $k(s) \rightarrow \infty$ ) results in high performance and high-perturbation rejection capabilities. The possibility of achieving such features have been widely studied, some very known results have been reported in [22-25].

The next step for dealing with the SIMO control problem consists in determining the effect of the main control loop over the secondary outputs. It is especially important to determine the effects that closing this control loop has over the stability and perturbation rejection characteristics of the secondary outputs. Let the transfer functions of interest be denoted by

$$
\begin{gathered}
c_{j, r}(s)=\frac{y_{j}(s)}{\operatorname{ref}(s)} \\
c_{j, p i}(s)=\frac{y_{j}(s)}{p_{e i}(s)},
\end{gathered}
$$




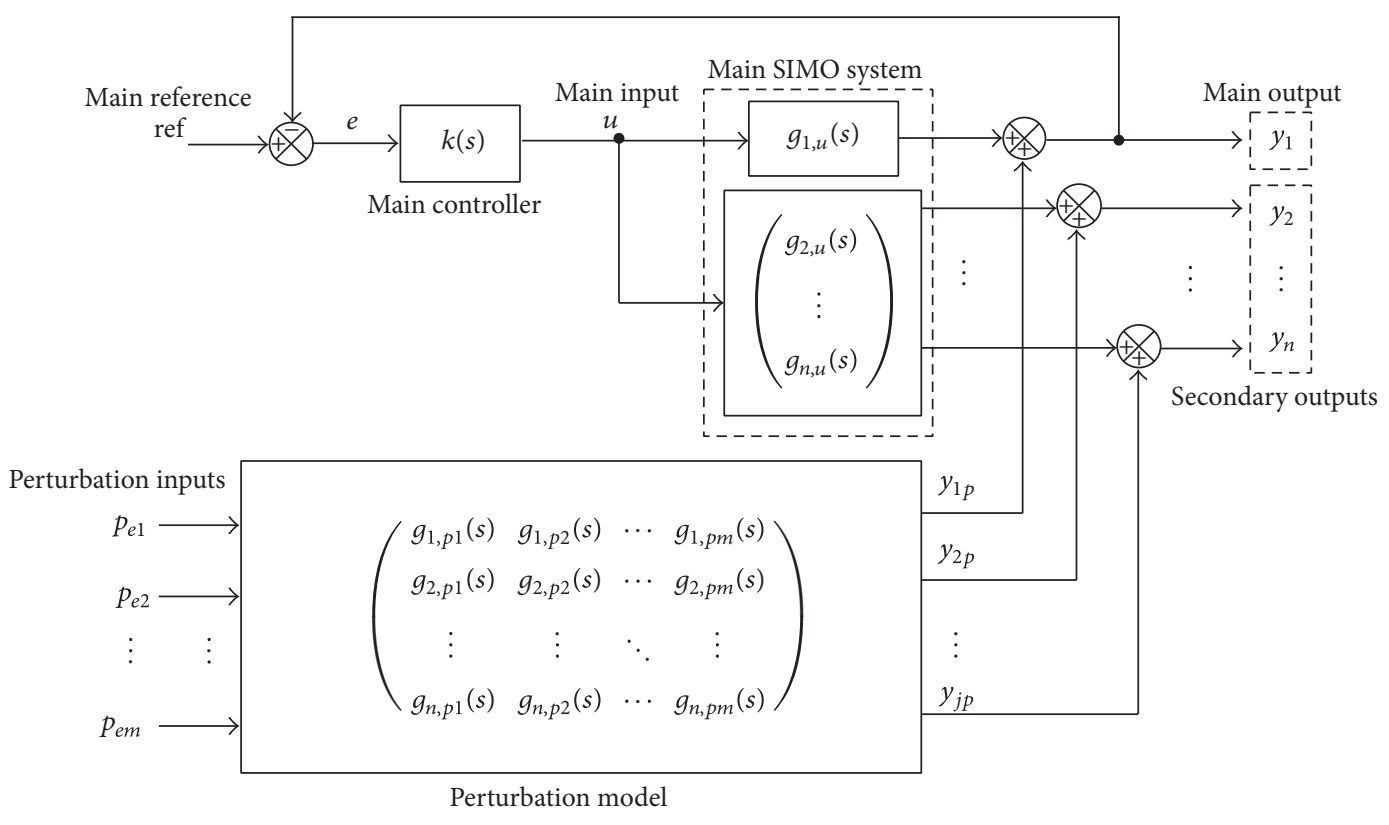

FIgURe 1: Perturbed SIMO control scheme.

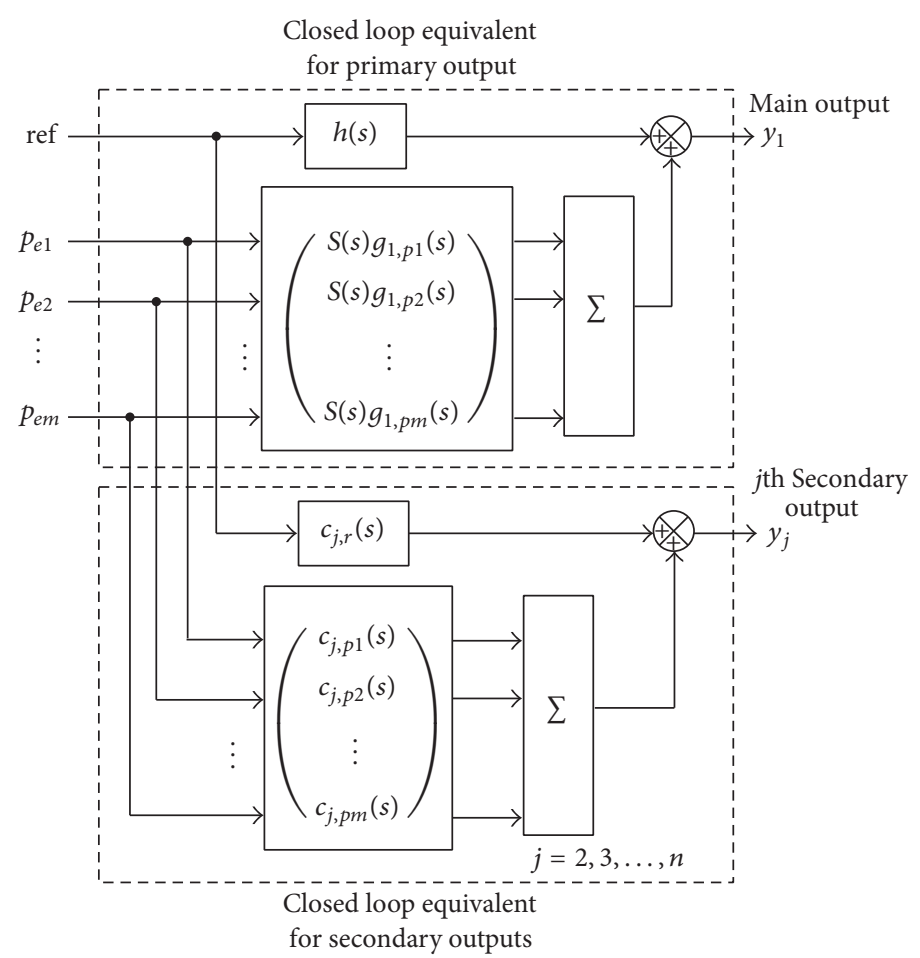

FIgURE 2: Closed-loop equivalent of the perturbed SIMO control system of Figure 1.

where $c_{j, r}(s)$ models the effect of the main reference over the secondary output $j$ and $c_{j, p i}(s)$ models the effect of the perturbation $i$ over output $j$. It is clear that

$$
y_{j}(s)=c_{j, r}(s) \operatorname{ref}(s)+\sum_{i=1}^{m} c_{j, p i}(s) p_{e i}(s) .
$$

According to (5) and (7) the scheme of Figure 1 can be restructured as shown in Figure 2 where the scalar transfer functions $h(s), S(s) g_{1, p i}(s), c_{j, r}(s)$, and $c_{j, p i}(s)$ model the effects of the main reference and the perturbations signals over all the outputs.

The control system stability depends on the stability of the set of transfer functions:

$$
\begin{aligned}
\mathbb{S} & =\left\{h(s), S(s) g_{1, p i}(s), c_{j, r}(s), c_{j, p i}(s): j \in\{2, n\}, i\right. \\
& \in\{1, m\}\} .
\end{aligned}
$$


On the other hand, the perturbation rejection capabilities are also defined by this set. Although it is easy to numerically (or even symbolically) calculate set (8) on each case, in the next section useful general conclusions will be derived. In particular, set $\mathbb{S}$ will be partitioned for analysis purposes as follows $\mathbb{S}=\left\{h(s), \mathbb{S}_{1}, \mathbb{S}_{2}, \mathbb{S}_{3}\right\}$ with $\mathbb{S}_{1}=\left\{S(s) g_{1, p i}(s): i \in\right.$ $\{1, m\}\}, \mathbb{S}_{2}=\left\{c_{j, r}(s): j \in\{2, n\}\right\}$ and $\mathbb{S}_{3}=\left\{c_{j, p i}(s): j \in\right.$ $\{2, n\}, i \in\{1, m\}\}$.

\section{Main Results}

The first element of set (8) (i.e., $h(s)$ ) is well-known and will not be studied further here. On the other hand, the main characteristics of $\mathbb{S}_{1}$ and $\mathbb{S}_{2}$ can be derived from typical SISO perturbation rejection analysis and will be presented with two brief lemmas. Finally, $\mathbb{S}_{3}$ is more involved and has not been studied thoroughly in the past. Therefore, the main results of this article deal with the characteristics of $\mathbb{S}_{3}$ and its significance for SIMO control design.

\section{Assumptions}

(i) Each element of matrix (1) represents a controllable and observable system. That is, there are no pole/zero cancellations on the individual transfer functions of (1). This is line with assuming that (1) is in an irreducible form.

(ii) There are no unstable zero/pole cancellations between the controller $h(s)$ and the plant. This constraint eases the dealing with internal stability whereas not really limiting the results in practice. Note that actual zero/pole cancellations are not really possible in practice.

Lemma 1. The set $\mathbb{S}_{1}=\left\{S(s) g_{1, p i}(s): i \in\{1, m\}\right\}$ is stable iff the closed-loop of the main control loop $h(s)$ is stable and the unstable modes of $g_{1, p i}(s)$ are included on the modes of $g_{1, u}(s) \forall i \in\{1, m\}$.

Proof. The proof is immediate since the stability of the sensitivity function of the main control loop is equivalent to the stability of the complementary sensitivity of the main control loop. The stability of transfer functions $g_{1, p i}(s)$ is an obvious requirement if no zero/pole cancellations between $S(s)$ and $g_{1, p i}(s)$ are introduced. On the other hand, if $g_{1, p i}(s)$ has unstable modes these modes are also roots of the denominator of $g_{1, p i}(s)$. Let $g_{1, u}(s)=n_{u}(s) / \bar{d}_{u}(s) \widetilde{d}_{u}(s)$ where $\bar{d}_{u}(s)$ contains the stable modes and $\widetilde{d}_{u}(s)$ is monic and contains the unstable modes; then $S(s)=\bar{d}_{u}(s) \widetilde{d}_{u}(s) k(s) /\left(\bar{d}_{u}(s) \widetilde{d}_{u}(s)+\right.$ $\left.k(s) n_{u}(s)\right)$; thus all the unstable modes appear on the numerator of $S(s)$. On the other hand, let $g_{1, p i}(s)=n_{p}(s) / \bar{d}_{p}(s) \widetilde{d}_{p}(s)$ where $\bar{d}_{u}(s)$ contains the stable modes and $\widetilde{d}_{p}(s)$ is monic and contains the unstable modes. Therefore, if all the unstable modes of $g_{1, p i}(s)$ are also modes of $g_{1, u}(s)$, then $\widetilde{d}_{u}(s)=$ $\widetilde{d}_{p}(s)$ and the roots corresponding to these modes will cancel in $S(s) g_{1, p i}(s)$ since they are factors of the numerator and the denominator, rendering $S(s) g_{1, p i}(s)$ input/output stable. Finally, there is no loss of internal stability because there is not loss of order in $S(s) g_{1, p i}(s)$ since the unstable modes lost from $g_{1, p i}(s)$ in the cancellation are fully contained in $g_{1, u}(s)$ as required by the lemma.

Discussion. Note that Lemma 1 refers to zero/pole cancellations of the roots corresponding to the same unstable modes. That is, it is not sufficient for the zero/pole cancellation to occur, it is necessary for it to involve the same mode; otherwise internal stability is lost. The consequences of Lemma 1 are well-known and impose obvious SISO requirements over the main control loop. In particular, similar conclusions may be reached through well-known perturbation rejection analysis. Therefore, due to lack of space further discussions in this regard will be omitted.

Lemma 2. The set $\mathbb{S}_{2}=\left\{c_{j, r}(s): j \in\{1, n\}\right\}$ is stable iff set $\mathbb{S}_{1}$ is stable and all the unstable modes of $g_{j, u}(s)$ are also modes of $g_{1, u}(s) \forall j \in\{2, n\}$.

Proof. First, when $j=1$ then $c_{j, r}(s)=h(s)$ whose stability is included on the stability of set $\mathbb{S}_{1}$. On the other cases a simple algebraic exercise reveals that $c_{j, r}(s)=h(s) g_{1, u}(s)^{-1} g_{j, u}(s)=$ $\left(k(s) /\left(1+k(s) g_{1, u}(s)\right)\right) g_{j, u}(s)$. The stability of $h(s) g_{1, u}(s)^{-1}$, also called controller complementary sensitivity [22], is equivalent to the stability of $h(s)$.

The rest of the proof is similar to that of Lemma 1 . Briefly, let $g_{1, u}(s)=n_{1}(s) / d_{1}(s)$ and $g_{j, u}(s)=n_{j}(s) / d_{j}(s)$; then $c_{j, r}(s)=\left(d_{1}(s) k(s) /\left(d_{1}(s)+k(s) n_{1}(s)\right)\right)\left(n_{j}(s) / d_{j}(s)\right)$. If $g_{j, u}(s)$ and $g_{1, u}(s)$ share unstable modes then the roots corresponding to those modes will cancel. However, in a similar fashion as in Lemma 1, the requirement is not only algebraic it is required for the cancellation to be of the same modes. Other unstable zero/pole cancellations cannot occur since $h(s) g_{1, u}(s)^{-1}$ is internally stable due to assumption (ii).

Discussion. The result of Lemma 2 is also straightforward. The effect of the main control loop reference over the secondary output $j$ is equal to the controller complementary sensitivity and the open-loop transfer function relating the main input to the secondary output. That is: $c_{j, r}(s)=h(s) g_{1, u}(s)^{-1} g_{j, u}(s)$. In addition, it is clear that stabilizing unstable plant modes via the main output will stabilize the same unstable modes for all outputs if they are observable/controllable with the main input/output pairing.

A basic result of the ICAD theory for $2 \times 2$ systems will be introduced next. This result is fundamental for the development of the main results of the article.

Lemma 3. Let a $2 \times 2$ diagonal control system given by

$$
\begin{aligned}
\left(\begin{array}{l}
y_{1}(s) \\
y_{2}(s)
\end{array}\right) & =\left(\begin{array}{ll}
g_{11}(s) & g_{12}(s) \\
g_{21}(s) & g_{22}(s)
\end{array}\right)\left(\begin{array}{l}
u_{1}(s) \\
u_{2}(s)
\end{array}\right) \\
& =Y(s) G(s) U(s) \\
\left(\begin{array}{l}
u_{1}(s) \\
u_{2}(s)
\end{array}\right) & =\left(\begin{array}{cc}
k_{1}(s) & 0 \\
0 & k_{2}(s)
\end{array}\right)\left(\begin{array}{l}
e_{1}(s) \\
e_{2}(s)
\end{array}\right)=U(s) \\
& =K(s) E(s)
\end{aligned}
$$



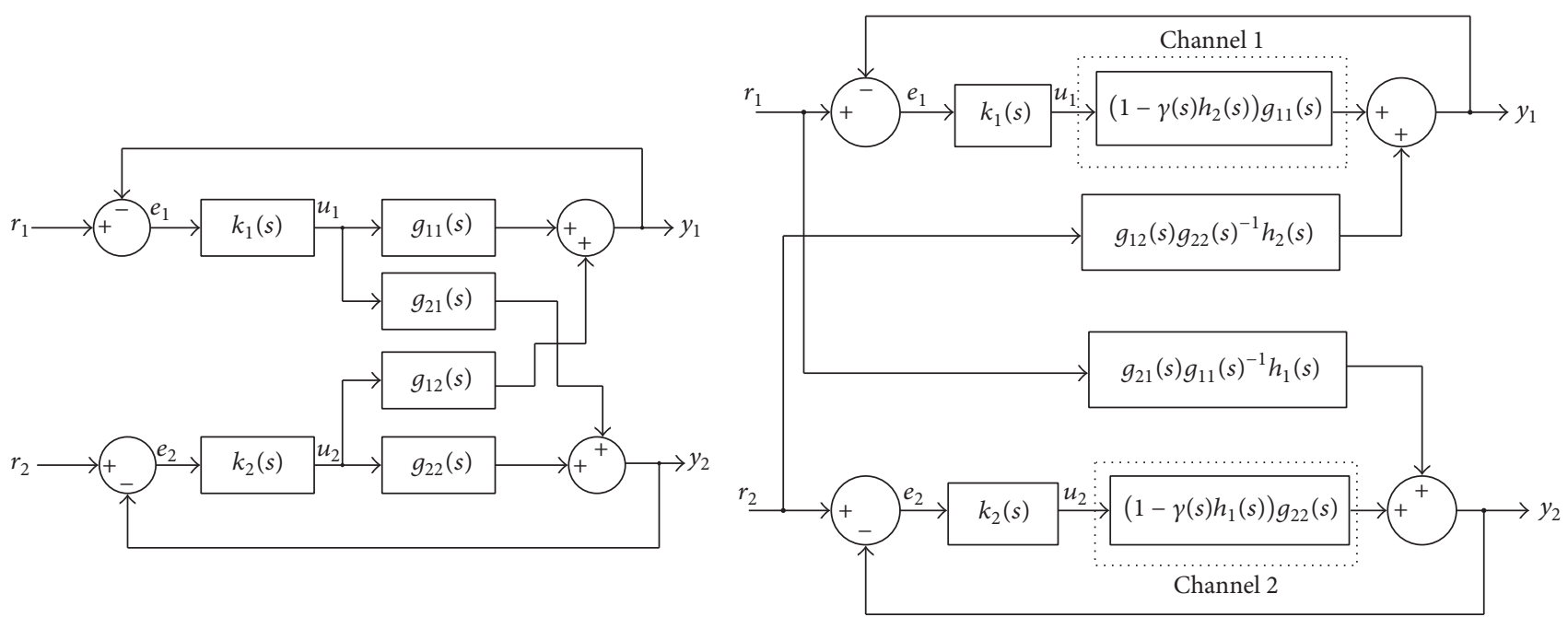

Figure 3: Multivariable $2 \times 2$ control system with a diagonal controller and the equivalent individual channels decomposition.

with $e_{i}(s)=r_{i}(s)-y_{i}(s)$. Then the output equations may be rewritten without assumptions or loss of information as

$$
\begin{aligned}
y_{i}(s)= & \frac{k_{i}(s) c_{i}(s)}{1+k_{i}(s) c_{i}(s)} r_{i}(s) \\
& +\frac{1}{1+k_{i}(s) c_{i}(s)} \frac{g_{i j}(s)}{g_{j j}(s)} h_{j}(s) r_{j}(s) \\
c_{i}(s)= & \frac{y_{i}(s)}{u_{i}(s)}=g_{i i}(s)\left(1-\gamma(s) h_{j}(s)\right) ; \\
h_{i}(s)= & \frac{k_{i}(s) g_{i i}(s)}{1+k_{i} g_{i i}(s)} \\
\gamma(s)= & \frac{g_{12}(s) g_{21}(s)}{g_{11}(s) g_{22}(s)}, i, j=1,2
\end{aligned}
$$

where $c_{i}(s)$ are called individual channels and $\gamma(s)$ is the Multivariable Structure Function (MSF).

The representation of the individual channel decomposition can be graphically represented as shown in Figure 3. The proof of Lemma 3 can be found in [17].

Discussion. There are many facts brought to light by Lemma 3. In particular the MSF can be very useful for evaluating the characteristics of multivariable systems. This function is inherent to the nature of the process and reveals important dynamical characteristics related to the existence of robust controllers satisfying arbitrary specifications. The main characteristics of the MSF are as follows [17, 21, 26]:

(i) It determines the cross-coupling characteristics of each input-output configuration. In particular, its magnitude quantifies the coupling between channels.

(ii) It has an interpretation in the frequency domain. (iii) The transmission zeros of $G(s)$ coincide with the roots of $1-\gamma(s)=\operatorname{det}[G(s)]=g_{11} g_{22}-g_{12} g_{21}$.

(iv) Its closeness to $(1,0)$ in the Nyquist plot indicates to what extent the plant structure (not necessarily its stability) is sensitive to uncertainty.

(v) It allows evaluating the robustness of decoupling controllers.

(vi) There is a close relationship between the MSF and the relative gain array.

One of the main advantages of this decomposition of $2 \times 2$ systems is that the multivariable response of any given output can be written as a scalar transfer function relating the complementary sensitivity of the individual channel equation $c_{i}(s)$ and the sensitivity of the same individual channel. This allows designing controller $k_{i}(s)$ as a traditional SISO control task with perturbation rejection specifications. Finally, an important aspect is that the individual channel $c_{i}(s)$ can also be used to model the open-loop input-output transfer function $y_{i}(s) / u_{i}(s)$ when output $y_{j}(s)$ is controlled via $u_{j}(s)$. In this setup the ICAD framework is suitable for studying the SIMO perturbation rejection problem.

It is known that there is a close relationship between the MSF and the transmission zeros of the system [17, 27]. However, this relationship has not been completely characterized. The next result further shows this relationship for $2 \times 2$ systems and serves as an accessory to the main result of this article. However, this result may also be helpful in other contexts.

Theorem 4. Let a controllable and observable $2 \times 2$ system with transfer function matrix (TFM) be $G_{a}(s)$ such that

$$
G_{a}(s)=\frac{\left(\begin{array}{ll}
n_{a 11}(s) & n_{a 12}(s) \\
n_{a 21}(s) & n_{a 22}(s)
\end{array}\right)}{d_{a}(s)},
$$

where $d_{a}(s)$ is the system poles polynomial obtained from the Smith-McMillan decomposition of $G_{a}(s)$. Then the MSF $\gamma_{a}(s)$ 
of $G_{a}(s)$ defined according to (9) and (13) is equal to $\gamma_{a}(s)=$ $n_{a 12}(s) n_{a 21}(s) / n_{a 22}(s) n_{a 11}(s)$ and the following is true:

$$
1-\gamma_{a}(s)=\frac{k_{\alpha} z_{a}(s) d_{a}(s)}{n_{a 11}(s) n_{a 22}(s)}
$$

where $z_{a}(s)$ is a polynomial which contains the transmission zeros of $G_{a}(s)$ and $k_{\alpha}$ is a real constant.

Proof. The Smith-McMillan decomposition of $G_{a}(s)$ yields $[28,29]$

$$
\operatorname{SM}_{G a}(s)=\left(\begin{array}{cc}
\frac{\operatorname{mcf}_{a}(s)}{d_{a}(s)} & 0 \\
0 & \frac{\left(n_{a 11}(s) n_{a 22}(s)-n_{a 12}(s) n_{a 21}(s)\right) /\left(\operatorname{mcf}_{a}(s) k_{\alpha}\right)}{d_{a}(s)}
\end{array}\right)=\left(\begin{array}{cc}
\operatorname{sm}_{1}(s) & 0 \\
0 & \operatorname{sm}_{2}(s)
\end{array}\right)
$$

where $\operatorname{mcf}_{a}(s)$ denotes the maximum monic common factor of the set $\left\{n_{a 11}(s), n_{a 22}(s), n_{a 12}(s), n_{a 21}(s)\right\}$ and $k_{\alpha}$ is a constant such that $\mathrm{sm}_{2}(s)$ is monic. The first part of the proof consists in showing that there cannot by zero/pole cancellations on $\operatorname{sm}_{1}(s)$. Consider the contrary that there is at least one zero/pole cancellation in $\operatorname{sm}_{1}(s)$. Without loss of generality let this factor be denoted by $\left(s+a_{0}\right)$. Since the numerator of $\operatorname{sm}_{1}(s)$ is the maximum common factor of all the numerators of $G_{a}(s)$ then it must be possible to write $G_{a}(s)=\left(s+a_{0}\right) \widetilde{G}_{a}(s)$. Recall that $G_{a}(s)$ has a common denominator equal to the denominator of $\operatorname{sm}_{1}(s)$. Then, since the denominator of both $G_{a}(s)$ and $\operatorname{sm}_{1}(s)$ is the system poles polynomial a zero/pole cancellation on $\operatorname{sm}_{1}(s)$ indicates the nonobservability/noncontrollability of the mode $\left(s+a_{0}\right)$ which contradicts the definition of $G_{a}(s)$.

The next part of the proof consists in showing that all the poles of $\mathrm{sm}_{2}(s)$ must cancel with some of the zeros of $\mathrm{sm}_{2}(s)$. This is easy to show recalling that the system poles polynomial of $G_{a}(s)$ is equal to the denominator of $\operatorname{sm}_{1}(s) \operatorname{sm}_{2}(s)$ after computing the zero-pole cancellations individually on $\operatorname{sm}_{1}(s)$ and $\mathrm{sm}_{2}(s)[28,29]$. Since there are no zero-pole cancellations on $\operatorname{sm}_{1}(s)$ then $d_{a}(s)$ must cancel in $\operatorname{sm}_{2}(s)$; otherwise the system poles polynomial would result different than $d_{a}(s)$ which is not possible from the definition of $G_{a}(s)$. This last result shows that $\operatorname{sm}_{2}(s)$ may be rewritten as $\operatorname{sm}_{2}(s)=$ $d_{a}(s) \widetilde{z}_{a}(s) / d_{a}(s)$ where $\widetilde{z}_{a}(s)$ is coprime with $d_{a}(s)$; thus the numerator of $\operatorname{sm}_{2}(s)$ is

$$
\frac{n_{a 11}(s) n_{a 22}(s)-n_{a 12}(s) n_{a 21}(s)}{\operatorname{mcf}_{a}(s) k_{\alpha}}=d_{a}(s) \widetilde{z}_{a}(s) .
$$

It is known that the transmission zeros polynomial of $G_{a}(s)$, denoted as $z_{a}(s)$, is equal to the numerator of $\operatorname{sm}_{1}(s) \mathrm{sm}_{2}(s)$ after computing the zero/pole cancellations individually on $\operatorname{sm}_{1}(s)$ and $\mathrm{sm}_{2}(s)[28,29]$. It was shown that there are no cancellations in $\operatorname{sm}_{1}(s)$ and after cancellations the numerator of $\operatorname{sm}_{2}(s)$ yields $\widetilde{z}_{a}(s)$; thus $z_{a}(s)=\operatorname{mcf}_{a}(s) \widetilde{z}_{a}(s)$. Considering this and (17) then

$$
n_{a 11}(s) n_{a 22}(s)-n_{a 12}(s) n_{a 21}(s)=k_{\alpha} z_{a}(s) d_{a}(s)
$$

Finally, it is easy to see that the MSF of $G_{a}(s)$, defined according to (9) and (13), yields $\gamma_{a}(s)=n_{a 12}(s) n_{a 21}(s) /$ $n_{a 22}(s) n_{a 11}(s)$ because of the common denominator of $G_{a}(s)$. Finally, considering this and (18) yields

$$
1-\gamma_{a}(s)=\frac{k_{\alpha} z_{a}(s) d_{a}(s)}{n_{a 11}(s) n_{a 22}(s)} .
$$

Using these basic results from ICAD the first main result is presented next. This result deals with the formal characteristics of transfer functions $c_{j, p i}(s)$ which model the effect of the perturbations over the secondary outputs when the main output is operated in closed-loop. First, it is opportune to introduce the following definition.

Definition 5. Let $\left(A_{j i}, B_{j i}, C_{j i}, D_{j i}\right)$ be a minimal realization of system $\left(\begin{array}{ccc}g_{1, u}(s) & g_{1, p i}(s) \\ g_{j, u}(s) & g_{j, p i}(s)\end{array}\right)$. The transfer function matrix (TFM) of this system is given by

$$
G_{j i}(s)=\frac{\left(\begin{array}{ll}
n_{1, u}(s) & n_{1, p i}(s) \\
n_{j, u}(s) & n_{j, p i}(s)
\end{array}\right)}{d_{j i}(s)}
$$

where $d_{j i}(s)$ is the characteristic polynomial of $A_{j i}$, that is, the system poles polynomial of $G_{j i}(s)$. This representation allows expressing, for instance, $g_{j, p i}(s)=n_{j, p i}(s) / d_{j i}(s)$.

Note that whereas all the elements of the SIMO system (1) are controllable and observable this condition does not necessarily apply for the elements of (20). That is, there may be zero-pole cancellations in the individual elements of (20). The next theorem deals with the stability and the structure of $c_{j, p i}(s)$.

The following two theorems present the main results of the article. Theorem 6 sets the formalization of the characteristics of $c_{j, p i}(s)$ and Theorem 7 shows the implications of these characteristics for the perturbation rejection properties of SIMO control systems.

Theorem 6. Each of the elements of the set $\mathbb{S}_{3}=\left\{c_{j, p i}(s): j \in\right.$ $\{1, n\}, i \in\{1, m\}\}$ is stable iffh $(s)$ is stable and transfer function $n_{1, u}(s) / d_{j i}(s)$ from Definition 5 has only stable nonobservable/noncontrollable modes (i.e., stable zero/pole cancellations 


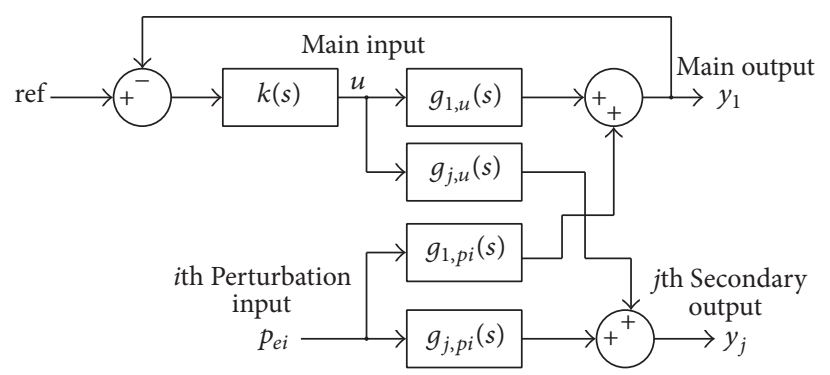

(a) Perturbed SIMO control subsystem

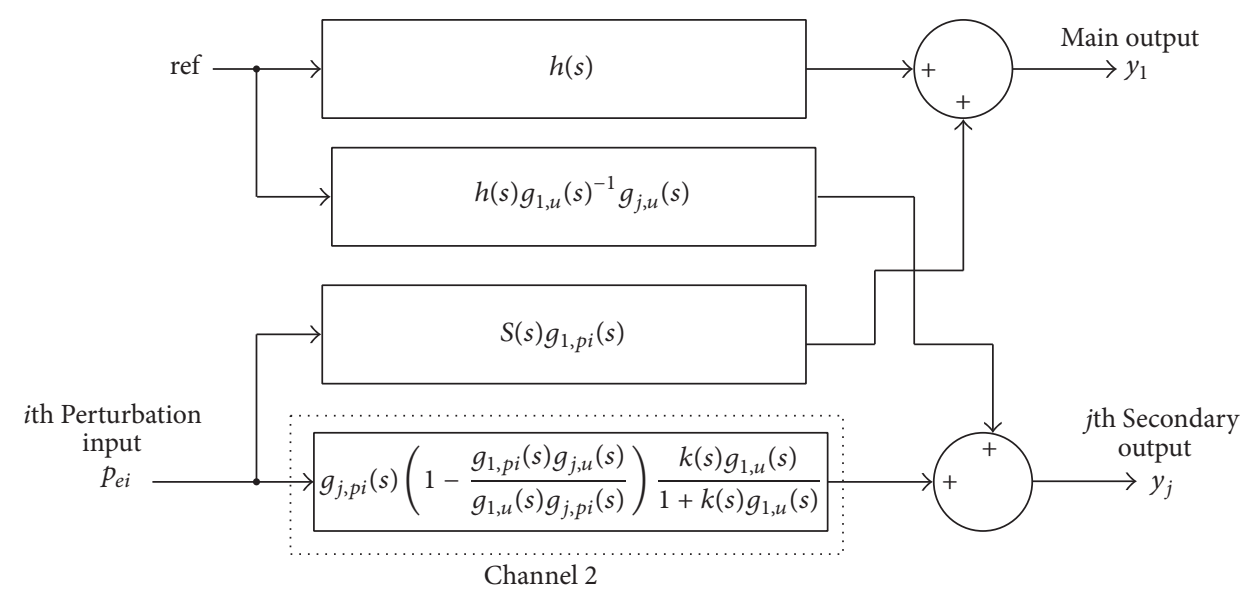

(b) Equivalent closed-loop subsystem

FIgURE 4: Decomposition of system (22).

between $n_{1, u}(s)$ and $\left.d_{j i}(s)\right)$. In particular:

$$
c_{j, p i}(s)=\frac{n_{j, p i}(s)+k_{\alpha} k(s) z_{j i}(s)}{d_{j i}(s)+k(s) n_{1, u}(s)}
$$

where $z_{j i}(s)$ is a polynomial containing the transmission zeros of $\operatorname{TFM}(20)$.

Proof. First, in order to establish the nature of transfer functions $c_{j, p i}(s)$ it is necessary to consider the following subsystem of the perturbed SIMO control system:

$$
\begin{aligned}
\left(\begin{array}{l}
y_{1}(s) \\
y_{j}(s)
\end{array}\right) & =\left(\begin{array}{ll}
g_{1, u}(s) & g_{1, p i}(s) \\
g_{j, u}(s) & g_{j, p i}(s)
\end{array}\right)\left(\begin{array}{l}
u_{1}(s) \\
p_{e i}(s)
\end{array}\right) \\
u_{1}(s) & =k(s) e(s) \\
e(s) & =\operatorname{ref}(s)-y_{1}(s) .
\end{aligned}
$$

System (22) adjusts partially to the typical $2 \times 2$ structure (Figure 3) with the difference that only one controller is used. It is easy to modify the basic structure of ICAD to accommodate system (22) by introducing the following modifications $k_{1}(s)=k(s), k_{2}(s)=0, u_{1}(s)=u(s), u_{2}(s)=$ $p_{e i}(s), r_{1}(s)=\operatorname{ref}(s), g_{11}(s)=g_{1, u}(s), g_{12}(s)=g_{1, p i}(s)$, $g_{21}(s)=g_{j, u}(s)$, and $g_{22}(s)=g_{j, p i}(s)$.
Then, according to Lemma 3:

$$
\begin{aligned}
& \frac{y_{j}(s)}{p_{e i}(s)}=c_{j, p i}(s) \\
& \quad=g_{j, p i}(s)\left(1-\frac{g_{1, p i}(s) g_{j, u}(s)}{g_{1, u}(s) g_{j, p i}(s)}\right) \frac{k(s) g_{1, u}(s)}{1+k(s) g_{1, u}(s)}
\end{aligned}
$$

That is, $c_{j, p i}(s)$ is equivalent to channel 2 of a typical $2 \times 2$ ICAD decomposition, as shown in Figure 4 . The other elements of this figure are obtained from Lemmas 1 and 2.

Recalling Definition 5 it is possible to write

$$
\begin{aligned}
& \frac{y_{j}(s)}{p_{e i}(s)}=c_{j, p i}(s)=\frac{n_{j, p i}(s)}{d_{j i}(s)}(1 \\
& \left.\quad-\frac{n_{1, p i}(s) n_{j, u}(s)}{n_{1, u}(s) n_{j, p i}(s)} \frac{k(s)\left(n_{1, u}(s) / d_{j i}(s)\right)}{1+k(s)\left(n_{1, u}(s) / d_{j i}(s)\right)}\right) .
\end{aligned}
$$
that

With some algebraic manipulation it is possible to show

$$
\frac{y_{j}(s)}{p_{e i}(s)}=c_{j, p i}(s)=\frac{n_{j, p i}(s)}{p_{j i}(s)}\left(\frac{n_{j, p i}(s) d_{j i}(s)+k(s)\left(n_{j, p i}(s) n_{1, u}(s)-n_{1, p i}(s) n_{j, u}(s)\right)}{n_{j, p i}(s) d_{j i}(s)+k(s) n_{1, u}(s) n_{j, p i}(s)}\right) .
$$


From Theorem 4 it follows that $n_{j, p i}(s) n_{1, u}(s)-$ $n_{1, p i}(s) n_{j, u}(s)=k_{\alpha} d_{j i}(s) z_{j i}(s)$; then:

$$
\begin{aligned}
& \frac{y_{j}(s)}{p_{e i}(s)}=c_{j, p i}(s) \\
& =\frac{n_{j, p i}(s)}{d_{j i}(s)}\left(\frac{n_{j, p i}(s) d_{j i}(s)+k_{\alpha} k(s) d_{j i}(s) z_{j i}(s)}{n_{j, p i}(s) d_{j i}(s)+k(s) n_{1, u}(s) n_{j, p i}(s)}\right) \\
& =\frac{n_{j, p i}(s)+k_{\alpha} k(s) z_{j i}(s)}{d_{j i}(s)+k(s) n_{1, u}(s)} .
\end{aligned}
$$

The stability of (26) can be elucidated according to the following. Let $k(s)=n_{k}(s) / d_{k}(s)$; then:

$$
c_{j, p i}(s)=\frac{d_{k}(s) n_{j, p i}(s)+k_{\alpha} n_{k}(s) z_{j i}(s)}{d_{k}(s) d_{j i}(s)+n_{k}(s) n_{1, u}(s)} .
$$

The denominator of (27) is similar to the denominator of $h(s)$ (3), which is the closed-loop of the main output, and $g_{1, u}(s)=n_{1, u}(s) / d_{j i}(s)$ by Definition 5. However, $n_{1, u}(s) / d_{j i}(s)$ may have zero/pole cancellations which are not present in $g_{1, u}(s)$ by assumption (i) (i.e., these cancellations are the modes which are not observable/controllable with the main input/output). Therefore the stability of (27) is equivalent to the stability $h(s)$ iff there are no unstable noncontrollable/nonobservable modes in $n_{1, u}(s) / d_{j i}(s)$.

In order to exploit fully the results from Theorem 6, the following elements of the perturbation rejection properties of the main and secondary outputs are defined:

(i) The main loop perturbation rejection (MPR) is equal to $S(s) g_{1, p i}(s)$. This is the perturbation rejection of the main control loop given by its sensitivity function and the perturbation model considering the $i$ th perturbation input.

(ii) The open-loop secondary perturbation rejection $(O S P R)$ is equal to $g_{j, p i}(s)$. This is the perturbation rejection of the secondary outputs when the system is operated in open-loop.

(iii) The closed-loop secondary perturbation rejection $(C S P R)$ is equal to $c_{j, p i}(s)$. This is the resulting perturbation rejection of the secondary outputs when the main control loop is used.

(iv) For simplicity, the notation $f(a) \rightarrow b$ will be introduced to indicate $\lim f(x)_{x \rightarrow a}=b$

(v) High-MPR at frequency $\omega_{0}$ is achieved when $g_{1, p i}\left(j \omega_{0}\right) S\left(j \omega_{0}\right) \rightarrow 0$ or equivalently $h\left(j \omega_{0}\right) \rightarrow 1$.

(vi) High-CSPR at frequency $\omega_{0}$ is achieved when $c_{j, p i}\left(j \omega_{0}\right) \rightarrow 0$.

When controlling only one output of a perturbed SIMO system such as (1) it is possible to stabilize the entire output vector iff the main output is stable and all the unstable modes are controllable and observable with the selected input/main output pair, that is, the zero-pole cancellation restriction of Theorem 6 . While this is obvious, using Theorem 6 it is easy to assess whether a particular \{perturbation, main output, secondary output\} triplet is stabilizable by constructing the TFM (20) and verifying that no unstable zero/pole cancellations occur in $n_{1, u}(s) / d_{j i}(s)$. This is akin to the Ackerman canonical form of system (1); however, it allows visualizing better which outputs are affected by each perturbation.

Theorem 6 also allows expressing the perturbation rejection capabilities of the secondary outputs as follows:

$$
c_{j, p i}(s)=\frac{S_{j i}(s)}{S(s)} g_{j, p i}(s),
$$

where $S(s)$ is the sensitivity of the main control loop and

$$
S_{j i}(s)=\frac{1}{1+k(s)\left(k_{\alpha} z_{j i}(s) / n_{j, p i}(s)\right)}
$$

can be interpreted as a virtual sensitivity function of the secondary control loop. Equation (28) introduces an intuitive relation between the perturbation rejection of the secondary outputs and the main control loop. For instance, it may be desirable to design $k(s)$ such that the virtual bandwidth of the closed-loop of $k(s)\left(k_{\alpha} z_{j i}(s) / n_{j, p i}(s)\right)$ is greater than the bandwidth of $h(s)$, so that $S_{j i}(s) / S(s)$ remains low for a certain frequency range.

One of the most revealing facts of Theorem 6 is that it allows introducing the following observation regarding the maximum achievable perturbation rejection of the secondary outputs. Let a SIMO system be defined as in Theorem 6; then there exist real positive constants $k_{1}>k_{2}$ such that the following is true:

$$
\begin{aligned}
& \left\|c_{j, p i}(j \omega)\right\| \approx\left\|\frac{k_{\alpha} z_{j i}(j \omega)}{n_{1, u}(j \omega)}\right\| \quad \forall \omega:\|k(j \omega)\|>k_{1} \\
& \left\|c_{j, p i}(j \omega)\right\| \approx\left\|g_{j, p i}(j \omega)\right\| \quad \forall \omega:\|k(j \omega)\|<k_{2} .
\end{aligned}
$$

To clarify this note that if $\|k(j \omega)\|$ is sufficiently large, then by (21) $\left\|c_{j, p i}(j \omega)\right\| \approx\left\|k_{\alpha} z_{j i}(j \omega) / n_{1, u}(j \omega)\right\|$. On the other hand, if $\|k(j \omega)\|$ is sufficiently small then $\left\|c_{j, p i}(j \omega)\right\| \approx$ $\left\|g_{j, p i}(j \omega)\right\|$. This may be interpreted as characterizing the limits of perturbation rejection on the secondary outputs by modifying the perturbation rejection characteristics of the main control loop. In particular:

(i) by increasing the MPR (i.e., increasing the gain of $k(s))$ the CSPR tends to the system $k_{\alpha} z_{j i}(s) / n_{1, u}(s)$

(ii) by decreasing the MPR (i.e., lowering the of $k(s)$ ) the CSPR tends to the OSPR, which is equal to $g_{j, p i}(j \omega)$.

In practical applications it would be desirable for a high level of perturbation rejection in the main input to be also indicative of a high level of perturbation rejection in the secondary outputs. In this sense, general conditions for having high-MPR and high-CSPR at the same time are presented next.

Theorem 7. If $c_{j, p i}(s)$ and $g_{j, p i}(s)$ are stable then the following conditions apply: $h\left(j \omega_{0}\right) \rightarrow 1 \wedge \gamma_{j i}\left(j \omega_{0}\right) \rightarrow 1 \Rightarrow c_{j, p i}\left(j \omega_{0}\right) \rightarrow$ 
0 and $h\left(j \omega_{0}\right) \rightarrow 1 \wedge c_{j, p i}\left(j \omega_{0}\right) \rightarrow 0 \wedge\left\|n_{j, p i}\left(j \omega_{0}\right)\right\| \neq 0 \Rightarrow$ $\gamma_{j i}\left(j \omega_{0}\right) \rightarrow 1$ where $\gamma_{j i}(s)=n_{1, p i}(s) n_{j, u}(s) / n_{1, u}(s) n_{j, p i}(s)$ and " $\wedge \vee$ " denote the logical operators "and or."

Proof. The first part of the proof of Theorem 7 consists on rewriting the perturbed SIMO problem in the typical ICAD structure, and therefore it is equivalent to the first part of the proof of Theorem 6 . The proof of $h\left(j \omega_{0}\right) \rightarrow 1 \wedge \gamma_{j i}\left(j \omega_{0}\right) \rightarrow$ $1 \Rightarrow c_{j i}\left(j \omega_{0}\right) \rightarrow 0$ is obtained by noticing $h\left(j \omega_{0}\right) \rightarrow$ $1 \wedge \gamma_{j i}\left(j \omega_{0}\right) \rightarrow 1 \Rightarrow\left(1-\gamma_{j i}\left(j \omega_{0}\right) h\left(j \omega_{0}\right)\right) \rightarrow 0$; therefore $h\left(j \omega_{0}\right) \rightarrow 1 \wedge \gamma_{j i}\left(j \omega_{0}\right) \rightarrow 1 \Rightarrow c_{j i}\left(j \omega_{0}\right) \rightarrow 0$ if $\left\|g_{j, p i}\left(j \omega_{0}\right)\right\| \rightarrow$ $\infty$ is false, which has to be because $\left\|g_{j, p i}\left(j \omega_{0}\right)\right\| \rightarrow \infty$ would imply that $g_{j, p i}(s)$ is critically stable contradicting the requirements. This completes the first part of the theorem.

For the second part note that $c_{j, p i}\left(j \omega_{0}\right) \rightarrow 0 \Rightarrow$ $h\left(j \omega_{0}\right) \rightarrow 1 \wedge \gamma_{j i}\left(j \omega_{0}\right) \rightarrow 1$ is not necessarily true because $c_{j, p i}\left(j \omega_{0}\right) \rightarrow 0$ can be obtained by other means than $h\left(j \omega_{0}\right) \rightarrow 1 \wedge \gamma_{j i}\left(j \omega_{0}\right) \rightarrow 1$. According to the proof of Theorem $6 c_{j, p i}\left(j \omega_{0}\right) \rightarrow 0 \Leftrightarrow n_{j, p i}\left(j \omega_{0}\right)+k_{\alpha} k\left(j \omega_{0}\right) z_{j i}\left(j \omega_{0}\right) \rightarrow$ $0 \vee\left\|d_{j i}\left(j \omega_{0}\right)+k\left(j \omega_{0}\right) n_{1, u}\left(j \omega_{0}\right)\right\| \rightarrow \infty$. Note that $\| d_{j i}\left(j \omega_{0}\right)+$ $k\left(j \omega_{0}\right) n_{1, u}\left(j \omega_{0}\right) \| \rightarrow \infty$ is not possible for real frequencies; therefore $c_{j, p i}\left(j \omega_{0}\right) \rightarrow 0 \Leftrightarrow n_{j, p i}\left(j \omega_{0}\right)+k_{\alpha} k\left(j \omega_{0}\right) z_{j i}\left(j \omega_{0}\right) \rightarrow$ 0 . Note that $n_{j, p i}(s)+k_{\alpha} k(s) z_{j i}(s)$ is the numerator of (26) which is zero if $n_{j, p i}\left(j \omega_{0}\right) \rightarrow 0$ or $\left(1-\gamma_{j i}\left(j \omega_{0}\right) h\left(j \omega_{0}\right)\right) \rightarrow 0$. If $k\left(j \omega_{0}\right) \rightarrow 0$; then $n_{j, p i}\left(j \omega_{0}\right) \rightarrow 0$ implies $c_{j, p i}\left(j \omega_{0}\right) \rightarrow 0$. That is, if the main control loop is deactivated the OSPR still can reach zero, but if $\left\|n_{j, p i}\left(j \omega_{0}\right)\right\| \neq 0$ then $c_{j, p i}\left(j \omega_{0}\right) \rightarrow 0 \Rightarrow$ $\left(1-\gamma_{j i}\left(j \omega_{0}\right) h\left(j \omega_{0}\right)\right) \rightarrow 0$. Combining both conditions yields $\left\|n_{j, p i}\left(j \omega_{0}\right)\right\| \neq 0 \wedge c_{j, p i}\left(j \omega_{0}\right) \rightarrow 0 \Rightarrow\left(1-\gamma_{j i}\left(j \omega_{0}\right) h\left(j \omega_{0}\right)\right) \rightarrow$ 0 . On the other hand $\left(1-\gamma_{j i}\left(j \omega_{0}\right) h\left(j \omega_{0}\right)\right) \rightarrow 0 \Rightarrow$ $h\left(j \omega_{0}\right) \rightarrow 1 \wedge \gamma_{j i}\left(j \omega_{0}\right) \rightarrow 1$ is not necessarily true; note that other combinations for $h\left(j \omega_{0}\right)$ and $\gamma_{j i}\left(j \omega_{0}\right)$ may still yield $\gamma_{j i}\left(j \omega_{0}\right) h\left(j \omega_{0}\right) \rightarrow 1$. However, $h\left(j \omega_{0}\right) \rightarrow 1 \wedge(1-$ $\left.\gamma_{j i}\left(j \omega_{0}\right) h\left(j \omega_{0}\right)\right) \rightarrow 0 \Rightarrow \gamma_{j i}\left(j \omega_{0}\right) \rightarrow 1$ is true. Finally, $h\left(j \omega_{0}\right) \rightarrow 1 \wedge c_{j, p i}\left(j \omega_{0}\right) \rightarrow 0 \wedge\left\|n_{j, p i}\left(j \omega_{0}\right)\right\| \neq 0 \Rightarrow \gamma_{j i}\left(j \omega_{0}\right) \rightarrow$ 1 .

This shows that it is possible to attain high-CSPR (i.e., $\left.c_{j, p i}\left(j \omega_{0}\right) \rightarrow 0\right)$ if $h i g h-M P R\left(\right.$ i.e., $\left.h\left(j \omega_{0}\right) \rightarrow 1\right)$ and $\gamma_{j i}\left(j \omega_{0}\right) \rightarrow$ 1. Note that there are other possibilities to achieve $c_{j i}\left(j \omega_{0}\right) \rightarrow$ 0 . However, these cases will not be covered in this article because while $h\left(j \omega_{0}\right) \rightarrow 1$ can be attained with good robustness margins through high controller gain, other cases imply either low perturbation rejection or low robustness in the main control loop. On the other hand, if the plant does not have high-OSPR (i.e., $\left.\left\|n_{j, p i}\left(j \omega_{0}\right)\right\| \neq 0\right)$ and high-MPR with high-CSPR are desired then it is necessary for the MSF to be equal to one (i.e., $\gamma_{j i}\left(j \omega_{0}\right) \rightarrow 1$ ).

Theorem 7 shows that in practice the possibility of attaining high-CSPR and high-MPR at the same time for a specific frequency can be evaluated by verifying if $\gamma_{j i}\left(j \omega_{0}\right) \rightarrow$ 1. In particular, it is possible to assess the closeness of $\gamma_{j i}(s)$ to the critical point $(1,0)$ for all the frequencies using classical Nyquist and Bode plots. Moreover, it is possible to calculate this closeness using classical phase and gain margins. An interesting observation is that $\gamma_{j i}\left(j \omega_{0}\right) \rightarrow 1$ typically implies $z_{j i}\left(j \omega_{0}\right)=0$ [27]. That is, simultaneous high-CSPR and high-MPR at frequency $\omega_{0}$ are possible if system (20) has transmission zeros on the imaginary axis, specifically at $j \omega_{0}$. Nonetheless care should be taken since $z_{j i}\left(j \omega_{0}\right) \rightarrow 0$ does not necessarily imply $\gamma_{j i}\left(j \omega_{0}\right) \rightarrow 1$. This fact is clear as it is possible for $n_{1, u}(s)$ and $z_{j i}(s)$ to have common factors. In this case $\gamma_{j i}\left(j \omega_{0}\right)$ may not tend to $(1,0)$ even if $z_{j i}\left(j \omega_{0}\right) \rightarrow 0$ due to cancellations of common factors. A further analysis of the cases when $\gamma_{j i}\left(j \omega_{0}\right) \rightarrow 1$ is out of the scope of this article.

In this context, it is pertinent to recall that the MSF has been historically used to measure the structural robustness of multivariable systems [8-14, 16-21]. As such, $\gamma\left(j \omega_{0}\right) \rightarrow 1$ is a sign of low structural robustness and it is undesired, since in that case the transmission zeros may change from minimum phase to nonminimum phase. In contrast, in the SIMO case, it is actually desirable that $\gamma_{i j}\left(j \omega_{0}\right) \rightarrow 1$ since it indicates that the system has interesting perturbation rejection characteristics.

\section{Illustrative Example}

In this section a very simple SIMO system is studied according to the results of the last section. The simplicity and straightforwardness of this particular system allow inferring several of its properties intuitively. It will be shown that these conclusions can be also derived using the theoretical results from the past section. Therefore, it is possible to establish a clear relationship between the theoretical and the intuitive notions of a basic SIMO system. The aim is to illustrate the mechanisms that will permit using the theoretical results with more complex systems that do not allow an intuitive analysis. Consider the three-tank system of Figure 5, where $q_{u}$ is the main system input flow, $h_{1}, h_{2}$, and $h_{3}$ are the height of the fluid in each tank, $A_{1}, A_{2}$, and $A_{3}$ model the capacity of each tank, $R_{1}, R_{2}$, and $R_{3}$ model the resistance to the flow of each valve, $q_{a}, q_{b}$, and $q_{c}$ are internal flows, and $q_{p 1}$ and $q_{p 2}$ are perturbation inputs. A simple linear approximation to this system is given by

$$
\begin{aligned}
\dot{h}_{1} & =\frac{q_{u}(t)-q_{a}(t)}{A_{1}} \\
\dot{h}_{2} & =\frac{q_{a}+q_{p 1}+q_{c}-q_{b}}{A_{2}} \\
\dot{h}_{3} & =\frac{q_{p 2}-q_{c}}{A_{3}} \\
q_{a}(t) & =\frac{h_{1}(t)-h_{2}(t)}{R_{1}} \\
q_{b}(t) & =\frac{h_{2}(t)}{R_{2}} \\
q_{c}(t) & =\frac{h_{3}(t)}{R_{3}} .
\end{aligned}
$$

System (31) can be considered a perturbed SIMO system with main input $q_{u}$, perturbation inputs $q_{p 1}, q_{p 2}$, and outputs $h_{1}, h_{2}$, and $h_{3}$. A space state representation of (31) is given by 


$$
\begin{aligned}
& \dot{x}=A x+B u \\
& y=C x \\
& A=\left(\begin{array}{ccc}
-\frac{1}{R_{1} A_{1}} & \frac{1}{R_{1} A_{1}} & 0 \\
\frac{1}{R_{1} A_{2}} & -\frac{1}{R_{2} A_{2}}-\frac{1}{R_{1} A_{2}} & \frac{1}{R_{3} A_{2}} \\
0 & 0 & -\frac{1}{R_{3} A_{3}}
\end{array}\right) \\
& B=\left(\begin{array}{ccc}
\frac{1}{A_{1}} & 0 & 0 \\
0 & \frac{1}{A_{2}} & 0 \\
0 & 0 & \frac{1}{A_{3}}
\end{array}\right) \\
& C=\left(\begin{array}{lll}
1 & 0 & 0 \\
0 & 1 & 0 \\
0 & 0 & 1
\end{array}\right) \\
& x=\left(\begin{array}{lll}
h_{1} & h_{2} & h_{3}
\end{array}\right)^{T} \\
& u=\left(\begin{array}{lll}
q_{u} & q_{p 1} & q_{p 2}
\end{array}\right)^{T} \text {. }
\end{aligned}
$$

The objective is to design a tracking control system for the main output $h_{1}$. In this case the control input is $q_{u}$ and the secondary outputs are $h_{2}$ and $h_{3}$. Before proceeding to the formal analysis of the system the following intuitive observations will be stated. The goal of the example is being able to assess these observations formally through the tools developed in the last section:

(a) It is possible to reject all perturbations over $h_{1}$ using the control input $q_{u}$ given that the dynamic of the third tank is stable.

(b) It will be possible to reject all perturbations over $h_{2}$ to a certain degree, depending mainly on the characteristics of $R_{1}$.

(c) If the dynamics of the second tank are unstable then stabilization is possible using the control input $q_{u}$ and measuring $h_{1}$.

(d) If the dynamic of the third tank is unstable it is not possible to stabilize the system.

(e) It is not possible to reject perturbations over $h_{3}$ using control input $q_{u}$.

(f) The output $h_{3}$ is only affected by the perturbation $q_{p 2}$.

Considering $A_{1}=1, A_{2}=2, A_{3}=1, R_{1}=2, R_{2}=1$, and $R_{3}=2$ an irreducible expression for the systems is

$$
\left(\begin{array}{l}
h_{1}(s) \\
h_{2}(s) \\
h_{3}(s)
\end{array}\right)=G_{T}(s)\left(\begin{array}{c}
q_{u}(s) \\
q_{p 1}(s) \\
q_{p 2}(s)
\end{array}\right)=\left(\begin{array}{ccc}
\frac{s+3 / 4}{(s+1 / 4)(s+1)} & \frac{1 / 4}{(s+1 / 4)(s+1)} & \frac{1 / 8}{(s+1 / 4)(s+1 / 2)(s+1)} \\
\frac{1 / 4}{(s+1 / 4)(s+1)} & \frac{s+1 / 2}{2(s+1 / 4)(s+1)} & \frac{1 / 4}{(s+1 / 4)(s+1)} \\
0 & 0 & \frac{1}{s+1 / 2}
\end{array}\right)\left(\begin{array}{c}
q_{u}(s) \\
q_{p 1}(s) \\
q_{p 2}(s)
\end{array}\right)
$$

Using the structure of (1) yields

$$
\begin{gathered}
\left(\begin{array}{l}
h_{1}(s) \\
h_{2}(s) \\
h_{3}(s)
\end{array}\right)=G_{T}(s)\left(\begin{array}{c}
q_{u}(s) \\
q_{p 1}(s) \\
q_{p 2}(s)
\end{array}\right) \\
=\left(\begin{array}{lll}
g_{1, u}(s) & g_{1, p 1}(s) & g_{1, p 2}(s) \\
g_{2, u}(s) & g_{2, p 1}(s) & g_{2, p 1}(s) \\
g_{3, u}(s) & g_{3, p 2}(s) & g_{3, p 2}(s)
\end{array}\right)\left(\begin{array}{l}
q_{u}(s) \\
q_{p 1}(s) \\
q_{p 2}(s)
\end{array}\right) .
\end{gathered}
$$

A direct application of Lemma 1 reveals that the stabilization of set $\mathbb{S}_{1}$ depends on the stability of the mode of $g_{3, p 2}(s)$ which is not present in $g_{1, u}(s)$ which is $s=-1 / 2$. Lemma 1 indicates that if this mode is unstable, then $\mathbb{S}_{1}$ would be unstable. This is the only mode which is nonobservable and noncontrollable with the main input-output pair, which is a mode corresponding to the third tank. This obvious result is in line with observation (d). On the other hand, applying
Lemma 2 shows that stabilization of the main output also implies stabilization of $\mathbb{S}_{2}$ because $\mathbb{S}_{1}$ was shown to be stable and $g_{2, u}(s), g_{3, u}(s)$ have no additional unstable modes which are not modes of $g_{1, u}(s)$. This is in line with observation (c). Another option for reaching these conclusions is using the Ackerman canonical form of (32).

A simple stabilizing controller for the main input is given by

$$
k_{T}(s)=\frac{1.17(s+3)}{s}
$$

The Bode plot of $k_{T}(s) g_{1, u}(s)$, presented in Figure 6, shows that the main control loop has a bandwidth around $2 \mathrm{rad} / \mathrm{s}$ and adequate robustness margins. This assesses the stability and transient characteristics of the first element of set $\mathbb{S}$, (8).

Using controller (35) Figure 7 summarizes the interactions modeled by sets $\mathbb{S}_{1}$ and $\mathbb{S}_{2}$. As was defined in Section 2, set $\mathbb{S}_{1}$ models the effect of the perturbations over the main output and the set $\mathbb{S}_{2}$ models the effect of the reference over all the secondary outputs. 


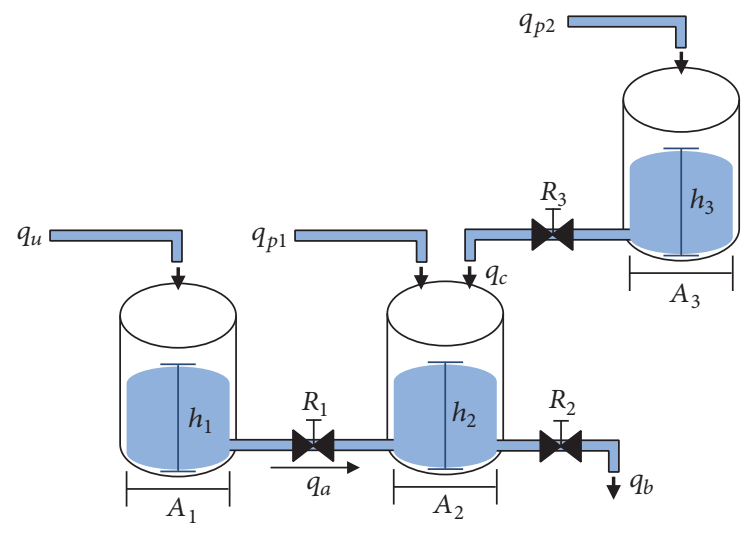

FIGURE 5: A perturbed three-tank SIMO system.

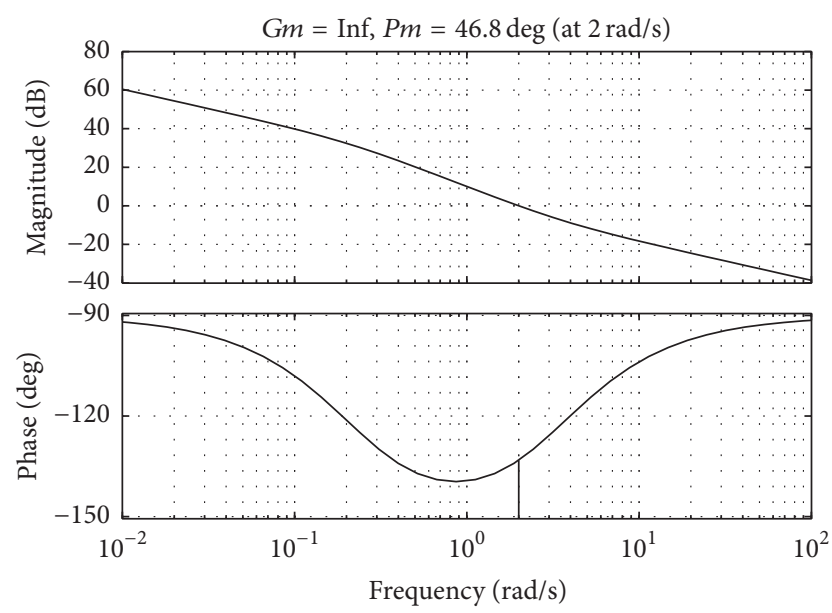

FIGURE 6: Bode plot of the open-loop main control loop $k_{T}(s) g_{1, u}(s)$.

In this case the effect of the reference over output $h_{3}$ is omitted because it is equal to zero. A more thorough discussion on these relationships is out of the scope of this article, as they may be fully characterized in terms of traditional classical perturbation rejection analysis. However, it is clear that an increment of the bandwidth of the main control loop by increasing the gain of controller $k_{T}(s)$ will decrease the effect of the perturbations over the main input. This is in line with observation (a).

In the following sections the main results of the article are used to elucidate the effects of the perturbations over the secondary outputs. The first step in the analysis consists in constructing system (22) for each secondary output and perturbation pair.

4.1. Case 1: Effect of $q_{p 1}$ over $h_{2}$. By considering secondary output $h_{2}$ and perturbation $p_{q 1}$, system (22) yields

$$
\left(\begin{array}{l}
h_{1}(s) \\
h_{2}(s)
\end{array}\right)=\frac{\left(\begin{array}{cc}
s+3 / 4 & 1 / 4 \\
1 / 4 & 0.5(s+1 / 2)
\end{array}\right)}{(s+1 / 4)(s+1)}\left(\begin{array}{c}
q_{u}(s) \\
q_{p 1}(s)
\end{array}\right) .
$$

In this case the MSF of system (36) results in $\gamma_{h 2, p 1}(s)=$ $0.125 /(s+3 / 4)(s+1 / 2)$; then, according to Theorem 4 , the transfer zeros polynomial of (36) is equal to $z_{1}(s)=1$ and $k_{\alpha}=1 / 2$. According to Theorem 7, it is not possible to have high-MPR and high-CSPR at any frequency because $\left\|\gamma_{h 2, p 1}(j \omega)\right\| \neq 1 \forall \omega \in \mathbb{R}$. That is, even if an infinite bandwidth controller for $h_{1}$ is used the perturbation rejection of $q_{p 1}$ over $h_{2}$ will be limited. In addition, according to Theorem 6 , imposing a high bandwidth control for $h_{1}$ will imply that the perturbation rejection for $h_{2}$ yields $h_{2}(s) / q_{p 1}(s)=$ $0.5 /(s+3 / 4)$ which is in line with the previous result. On the other hand the open-loop perturbation rejection for $h_{2}$ is equal to $h_{2}(s) / q_{p 1}(s)=1 / 2(s+1 / 2)$. Finally, as the first element of (36) does not have zero/pole cancellations all the modes contained in (36) are stabilized with the main control loop, and according to Theorem 6 , the perturbation dynamics will also be stable. This is in line with observation (c).

Figure 8 presents the Bode plot of the $\gamma_{h 2, p 1}(s)$; the closeness of this plot to the point $(1,0)$ assesses the possibility of achieving high-MPR and high-CSPR. Therefore, although it is clear that this is not possible at any frequency, at lower frequencies better CSPR could be expected by closing the main control loop. Any other remaining perturbation rejection in the secondary output will come directly from the OSPR. This indicates that if a bandwidth greater than $1 \mathrm{rad} / \mathrm{s}$ (i.e., the frequency when the MSF reaches around $-20 \mathrm{~dB}$ ) is used for the main output then the perturbation rejection of output $h_{2}$ will be near to its maximum. That is, no significant further reduction can be achieved by increasing the main output bandwidth over $1 \mathrm{rad} / \mathrm{s}$ since the magnitude of $\gamma_{h 2, p 1}(s)$ decrements at higher frequencies. Recalling Figure 6 , then controller (35) achieved sufficient bandwidth and no further increment of its bandwidth is necessary for increasing the CSPR.

Figure 9 shows the magnitude Bode diagrams of (1) the CSPR when high-MPR is considered, (2) the CSPR when controller (35) is used, and (3) when the main output is operated in open-loop (i.e., the OSPR). In this figure the CSPR using controller (35) and when high-MPR is considered are almost the same as predicted by the analysis of the MSF of Figure 8 . That is, by closing the main control loop, the perturbation rejection of the secondary output is improved, as long as the MSF is close to $(1,0)$.

The time response of $h_{2}$ when $q_{p 1}$ is a step signal is presented in Figure 10 for the same cases of Figure 9. This confirms the observations of the frequency domain analysis.

In order to explore further the effect of main control loop bandwidth over the CSPR the following controllers for the main output are considered:

$$
\begin{aligned}
k_{T_{\mathrm{LB}}}(s) & =\frac{2.5 \times 10^{-4}}{s(s+0.03)} \\
k_{T_{\mathrm{HB}}}(s) & =180 \frac{(s+150)}{s} .
\end{aligned}
$$

In this case $k_{T_{\mathrm{LB}}}(s)$ and $k_{T_{\mathrm{HB}}}(s)$ achieve bandwidths of $0.02 \mathrm{rad} / \mathrm{s}$ and $200 \mathrm{rad} / \mathrm{s}$, respectively, with similar robustness margins compared to (35). The Bode plots of the CSPR using both controllers and the OSPR are shown in Figure 11. This 

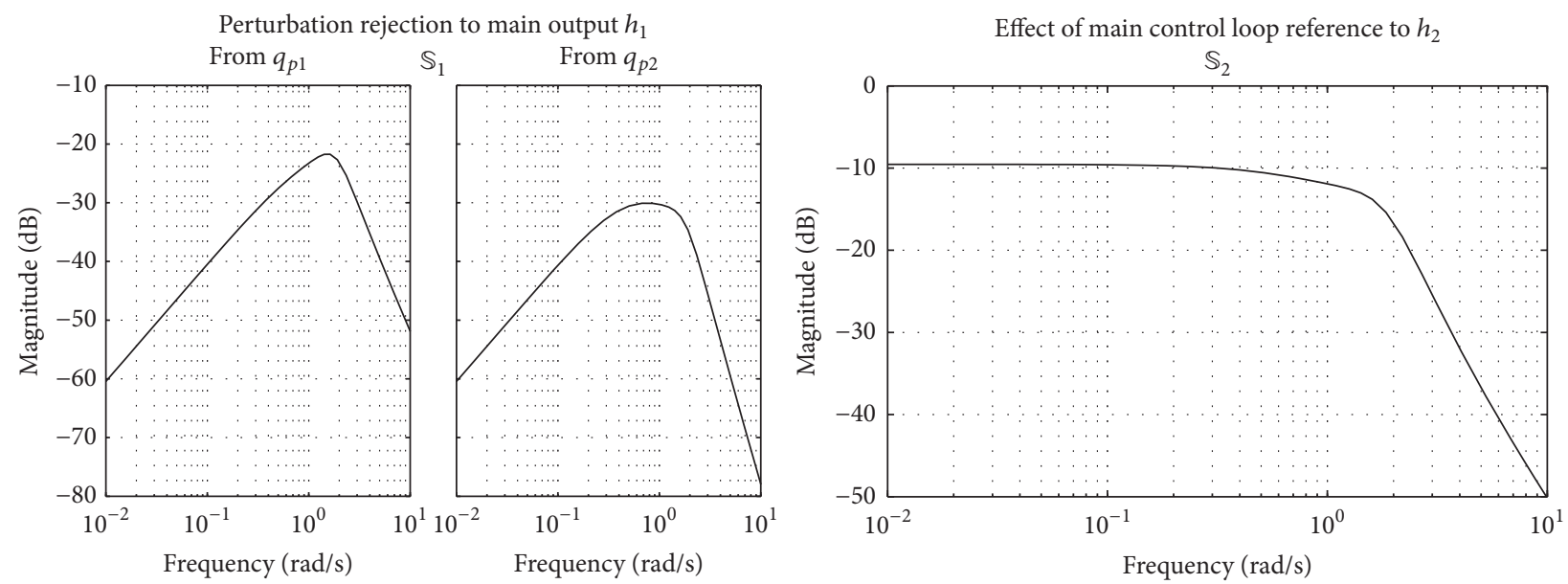

FIGURE 7: Frequency response of the nonzero elements of sets $\mathbb{S}_{1}$ and $\mathbb{S}_{2}$.

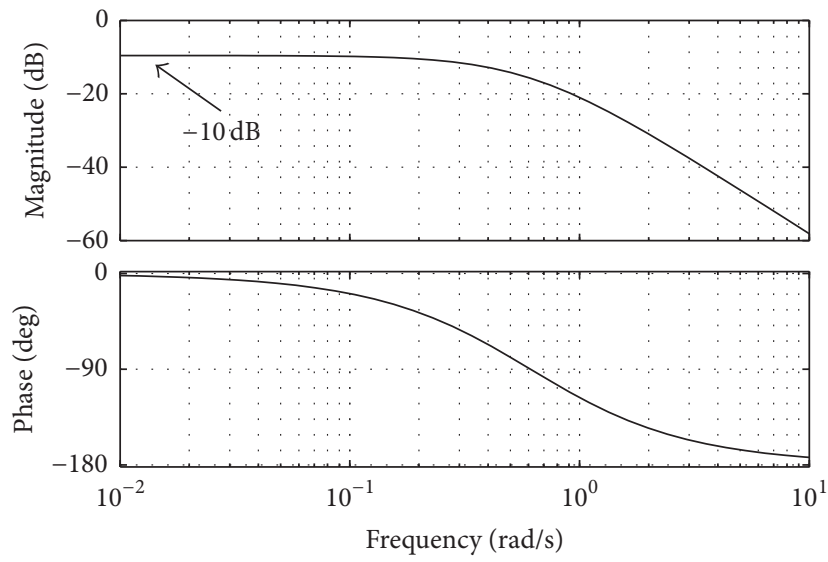

FIGURE 8: Bode Plot of $\gamma_{h 2, p 1}(s)$.

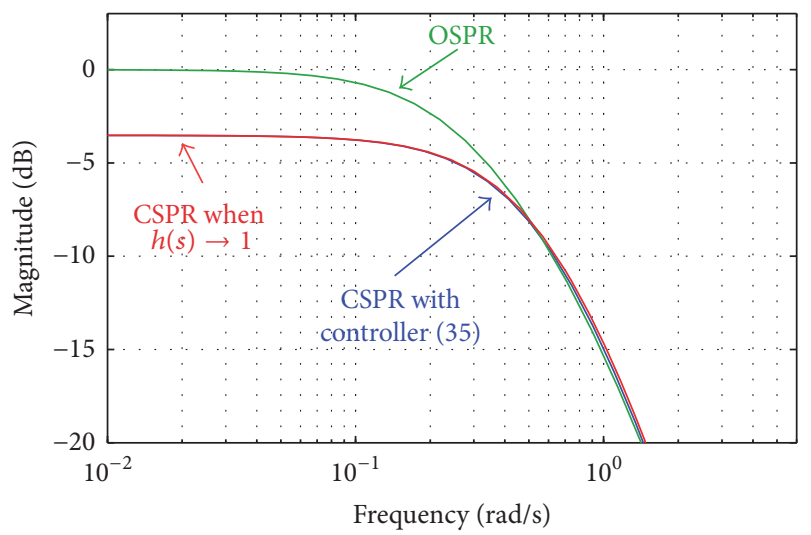

FIGURE 9: Bode plot of $h_{2}(s) / q_{p 1}(s)$ in several cases.

figure demonstrates that a lower bandwidth in the main output decreases the CSPR whereas a higher bandwidth yields no benefit compared with controller (35). In the low bandwidth case the figure also shows that when the main

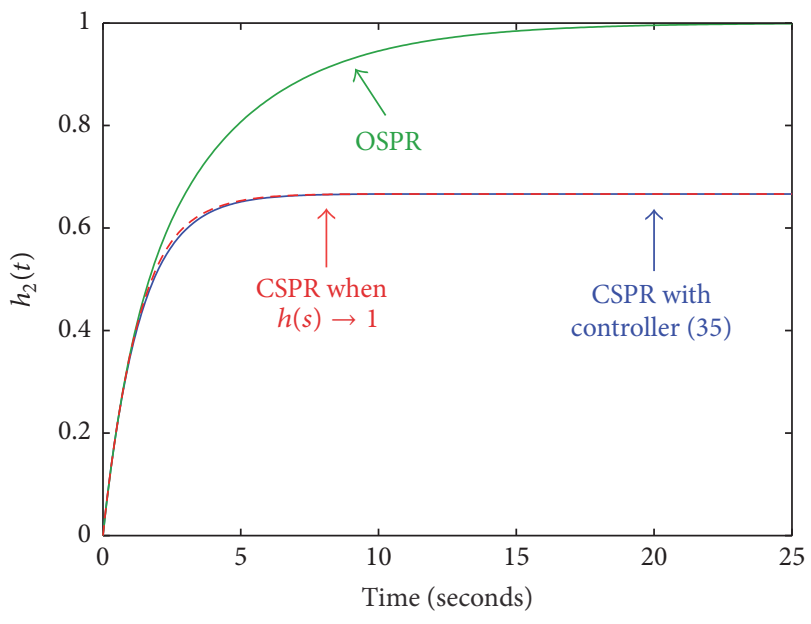

FIGURE 10: Time response of $h_{2}$ when $q_{p 1}$ is a step signal.

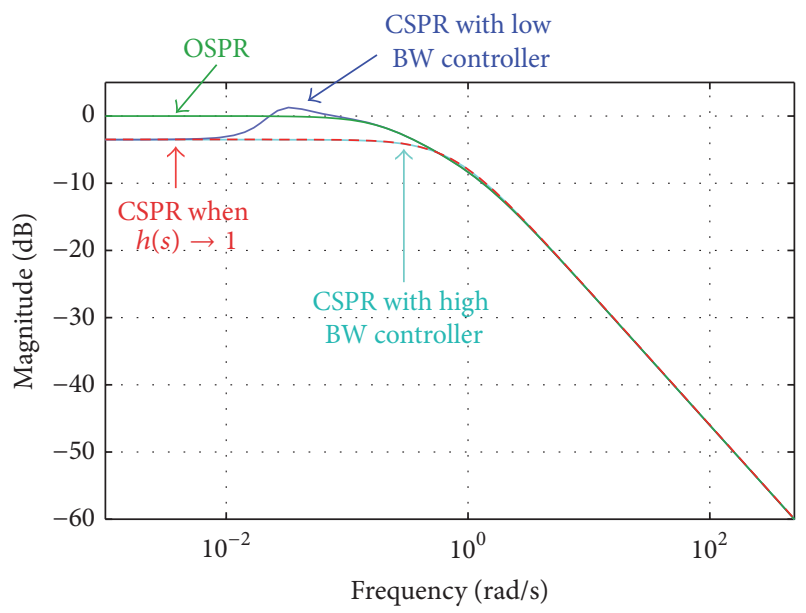

FIGURE 11: Bode plot of $h_{2}(s) / q_{p 1}(s)$ considering low and high bandwidth $(B W)$ controllers for the main output. 
output operates with high open-loop gain (i.e., at frequencies lower than $0.02 \mathrm{rad} / \mathrm{s}$ ) and with low open-loop gain (i.e., at frequencies higher than $0.02 \mathrm{rad} / \mathrm{s}$ ) the resulting CSPR can be described by (1) the CSPR when high-MPR is attained and (2) the OSPR, respectively. On the other hand, when the main output has mid-open-loop gain (i.e., close to $0 \mathrm{~dB}$ or close to $0.02 \mathrm{rad} / \mathrm{s}$ ) the resulting CSPR increases. This is in line with the discussion of Theorem 7 . That is, for attaining highCSPR it is recommended to aim for high open-loop gain in the main output (i.e., $h\left(j \omega_{0}\right) \rightarrow 1$ ) when $\gamma_{h 2, p 1}\left(j \omega_{0}\right)$ is near $(1,0)$. Otherwise, the effects of high sensitivity around the cross-over frequency will introduce further uncertainty in the resulting CSPR.

It is also possible to see how the perturbation rejection of the secondary outputs can be manipulated through the bandwidth of $h(s)$. The designer can choose either the OSPR or the CSPR when $h(s) \rightarrow 1$ by decreasing or increasing the open-loop gain of the main control loop. This is in line with the discussion regarding (30).

The results can also be corroborated in the time domain considering the step response of $h_{2}$ when $q_{p 1}$ is a step signal considering the controllers (37), which is presented in Figure 12. This figure confirms that increasing the bandwidth further does not provide further reduction of the CSPR. On the other hand, decreasing the bandwidth introduces a midfrequency mode with similar amplitude compared to the OSPR while conserving the same level of low frequency CSRP compared to the higher bandwidth controllers.

4.2. Case 2: Effect of $q_{p 2}$ over $h_{2}$. The system comprising the relevant variables yields, according to (22):

$$
\left(\begin{array}{l}
h_{1}(s) \\
h_{2}(s)
\end{array}\right)=\frac{\left(\begin{array}{cc}
(s+3 / 4)(s+1 / 2) & 1 / 8 \\
0.25(s+1 / 2) & 0.25(s+1 / 2)
\end{array}\right)}{(s+1 / 4)(s+1 / 2)(s+1)}\left(\begin{array}{c}
q_{u}(s) \\
q_{p 2}(s)
\end{array}\right) .
$$

The MSF of this system results in $\gamma_{h 2 p_{2}}(s)=0.125 /(s+$ $3 / 4)(s+1 / 2)$, which is equal to the previous case. Therefore, the frequency characteristics of the perturbation rejection are similar to that of the last case and will not be explored further.

On the other hand, the SM form reveals that system (38) has no transmission zeros. Calculating (21) for this output and perturbation configuration yields $h_{2}(s) / q_{p 2}(s)=0.25 /(s+$ $1 / 2)(s+3 / 4)$. This case is interesting as the calculation of the actual perturbation rejection using controller (36) results in

$$
\frac{h_{2}(s)}{q_{p 2}(s)}=\frac{0.25\left(s^{2}+1.67 s+3.51\right)}{(s+1 / 2)(s+0.78)\left(s^{2}+1.63 s+3.35\right)} \text {. }
$$

It is clear that the mode with pole $s=-1 / 2$ was not affected by the main control loop. This is expected since this mode is nonobservable/noncontrollable using the main control loop. According to the proof of Theorem 6 this results in the zero/pole cancellation observed in the first element of matrix (38). In the case of an unstable mode it would be impossible to stabilize the perturbation dynamic. This is in line with observations (c) and (d).

4.3. Case 3: Effect of $q_{p 1}$ and $q_{p 2}$ over $h_{3}$. The secondary output $h_{3}$ is decoupled from the main output. That is, while this variable has effect over the main output, the main input does not have effect over $h_{3}$. Therefore an intuitive conclusion is that perturbation rejection over this variable using the main control loop is not possible, that is, observation (e). This can be further validated by constructing the corresponding TFM according to (22):

$$
\begin{aligned}
\left(\begin{array}{l}
h_{1}(s) \\
h_{3}(s)
\end{array}\right) & =\frac{\left(\begin{array}{c}
(s+3 / 4)(s+1 / 2) \\
0
\end{array}\right)}{(s+1 / 4)(s+1 / 2)(s+1)}\left(\begin{array}{c}
q_{u}(s) \\
q_{p 1}(s)
\end{array}\right) \\
\left(\begin{array}{l}
h_{1}(s) \\
h_{3}(s)
\end{array}\right) & =\frac{\left(\begin{array}{cc}
(s+3 / 4)(s+1 / 2) & 1 / 8 \\
0 & (s+1 / 4)(s+1)
\end{array}\right)}{(s+1 / 4)(s+1 / 2)(s+1)}\left(\begin{array}{c}
q_{u}(s) \\
q_{p 2}(s)
\end{array}\right) .
\end{aligned}
$$

In both cases the MSF results equal to zero; therefore there cannot be CSPR. This leaves only the OSPR.

It is possible to confirm that Theorem 6 correctly predicts this for both cases. In the first case $h_{3}(s) / q_{p 1}(s)=0$ according to (21) which properly indicates that $h_{3}$ is not affected by $q_{p 1}$. In the other case $h_{3}(s) / q_{p 2}(s)=1 /(s+1 / 2)$ which confirms that the third tank is only affected in open-loop by $q_{p 2}$. These conclusions are in line with observations (e) and (f). Thus all the intuitive observations are now confirmed and extended.

4.4. Further Discussion on the Interpretation of the MSF for CSPR. In the previous cases the limits for the CSRP were analyzed for the example at hand. Although these limits may be studied via Theorem 6 , an additional tool is available in the form of Theorem 7 with the MSF.

According to Theorem 7, $\gamma\left(j \omega_{0}\right) \rightarrow 1$ defines the possibility of achieving high-CSPR by increasing the performance of the main control loop. In the previous cases the MSF (Figure 8) showed the CSPR is limited to a mild perturbation rejection in the lower frequencies up to around $1 \mathrm{rad} / \mathrm{s}$ and negligible at higher frequencies; this was confirmed later (Figures 9 and 10). Consider now the same system with different parameters $A_{1}=1, A_{2}=2, A_{3}=1, R_{1}=0.05$, $R_{2}=1$, and $R_{3}=2$. The main change is the considerable reduction of $R_{1}$. In this case the Bode plot of the MSF $\gamma_{h 2, p 1}(s)$ and $\gamma_{h 2, p 2}(s)$ (both are the equal) is shown in Figure 13.

This figure indicates that the system has improved CSPR characteristics with the new parameters. In particular, the $\gamma_{h 2, p 1}(s)$ is closer to the point $(1,0)$ for a wider frequency band. In order to exploit all the available CSPR the main control loop requires a bandwidth of at least $40 \mathrm{rad} / \mathrm{s}$. This fact is also obvious physically since reducing the restriction between tanks 1 and 2 (i.e., $R_{1}$ ) will clearly result in better perturbation rejection to $h_{2}$ by controlling $h_{1}$.

Controller (41) is a stabilizing controller with the same bandwidth (2 rad/s) and robustness for the main output as controller (35) was for the previous example (Figure 14).

$$
k_{T a}(s)=3 \frac{(s+3.5)}{s} .
$$

On the other hand controller (42) yields a bandwidth of $40 \mathrm{rad} / \mathrm{s}$ and the same robustness characteristics for the main control loop.

$$
k_{T b}(s)=20 \frac{(s+88)}{s} .
$$




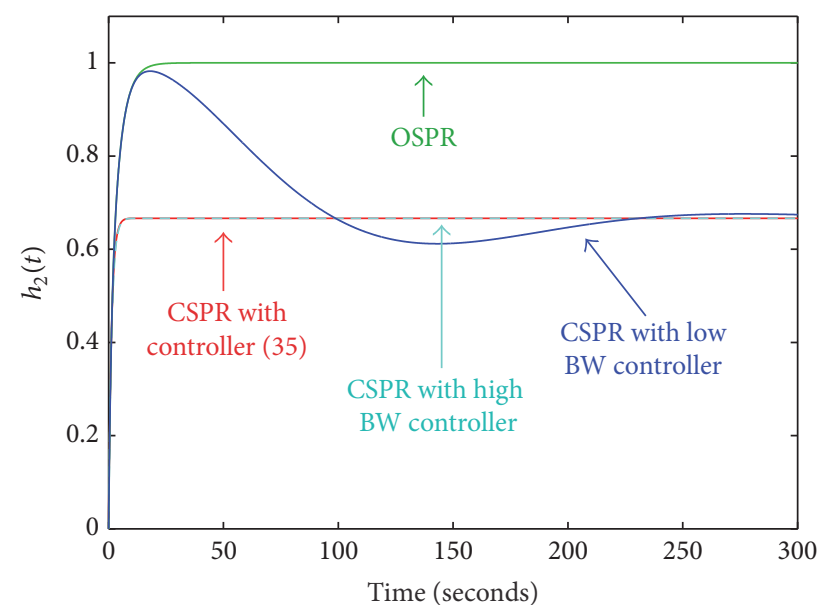

FIGURE 12: Time response of $h_{2}$ when $q_{p 1}$ is a step signal considering controllers of different bandwidth for the main output.
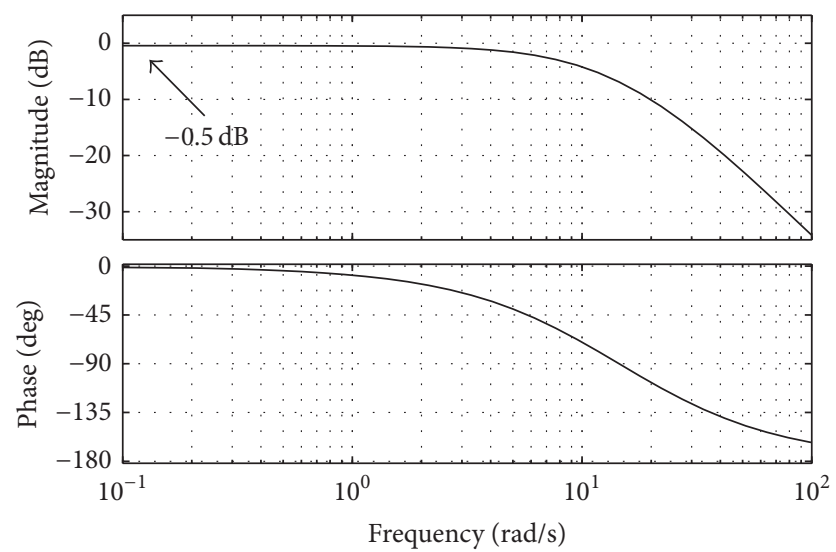

FIGURE 13: Bode plot of $\gamma_{h 2, p 1}(s)$ and $\gamma_{h 2, p 2}(s)$ considering a new set of system parameters.

According to the previous discussion and recalling that the MSF $\gamma_{h 2, p 1}(s)$ and $\gamma_{h 2, p 2}(s)$ are equal then the CSPR from perturbation inputs $q_{p 1}$ and $q_{p 2}$ will be reduced near to its minimum possible with controller (42) while controller (41) will have a CSPR close to the OSPR for frequencies between $2 \mathrm{rad} / \mathrm{s}$ and $40 \mathrm{rad} / \mathrm{s}$. Moreover, the resulting CSPR will be lower due to the fact that $\gamma_{h 2, p 1}(s)$ and $\gamma_{h 2, p 2}(s)$ are closer to $(1,0)$.

Figure 15 presents the magnitude Bode plots of $h_{2}(s) /$ $q_{p 1}(s)$ and $h_{2}(s) / q_{p 2}(s)$ considering the new system parameters and controllers (41)-(42), confirming all of the previous deductions. A comparison between $h_{2}(s) / q_{p 1}(s)$ and $h_{2}(s) / q_{p 2}(s)$ also shows the effect that the differences in OSPR have in the resulting CSPR. Note that the OSPR of $h_{2}(s) / q_{p 2}(s)$ has a stepper slope than that of $h_{2}(s) / q_{p 1}(s)$. This is due to (38) being of greater degree than (36).

Finally, a complete assessment of the time domain responses for all the perturbation rejection elements of the system is presented in Figure 16 considering the new systems parameters and controllers (41) and (42). The responses show that (1) for $h_{1}$ the perturbation rejection can be increased

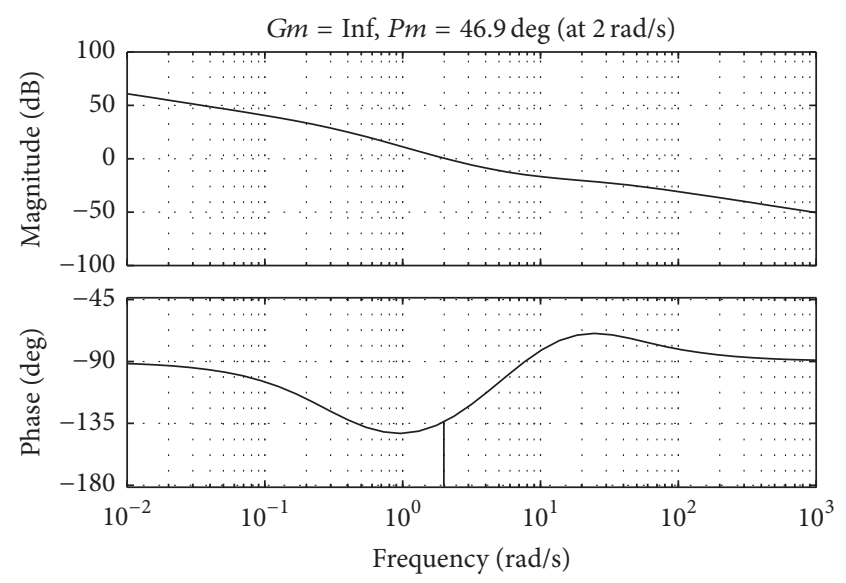

FIGURE 14: Bode plot of $k_{T a}(s) h_{1}(s) / q_{u}(s)$, the open-loop main control loop considering the new system parameters and controller (41).

arbitrarily by increasing the main control loop bandwidth, (2) for $h_{2}$ the perturbation rejection can be increased by increasing the main control loop bandwidth but it is limited by the structural properties of the system, and (3) for $h_{3}$ the perturbation rejection cannot be modified by the main control loop. All cases have been covered and predicted accordingly.

\section{Conclusions}

The multiple perturbations SIMO control case where a main output-feedback control is imposed was fully analyzed, and the resulting control system was segmented according an intuitive classification.

According to this classification, the stability of the different subsystems was formally defined. This classification of the internal control loops results in a better understanding of the eventual instability mechanisms of these control systems.

Particular attention was paid to the perturbation rejection characteristics of the resulting control system. The relationship between the frequency response characteristics of the main control loop with the perturbation rejection capabilities of the secondary outputs was fully analyzed.

This analysis resulted in several general conclusions which help the control designer in understanding better the nature of the process and making better design decisions. In particular, the article explores and formally characterizes the possibility of improving the perturbation rejection of the secondary outputs by increasing the bandwidth of the main control loop.

The results are first presented formally and then their relevance is fully explained through an intuitive case study. In the example it is shown how it is possible to integrate the main theoretical results with an intuitive understanding of SIMO control systems.

The use of classical frequency analysis tools allows the use of the results within an engineering context, improving their applicability with other widespread analysis tools, such as $H_{\infty}$. 

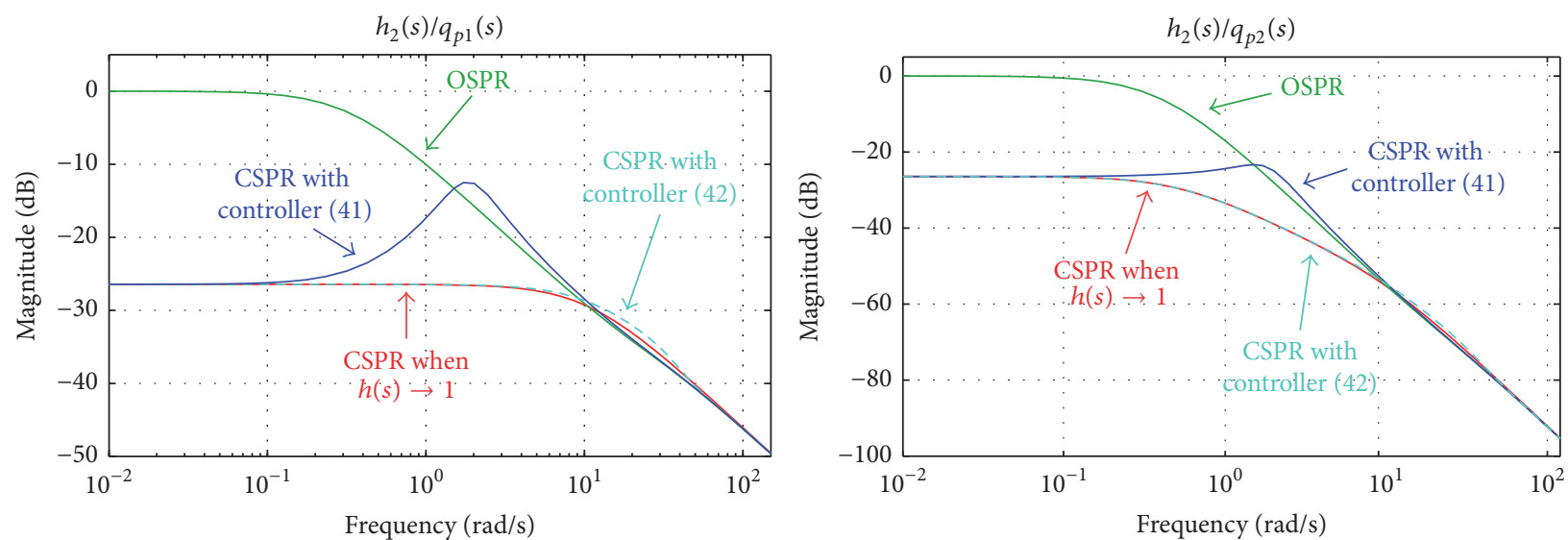

FIGURE 15: Bode plots of $h_{2}(s) / q_{p 1}(s)$ and $h_{2}(s) / q_{p 2}(s)$ considering the new system parameters and controllers (41)-(42).

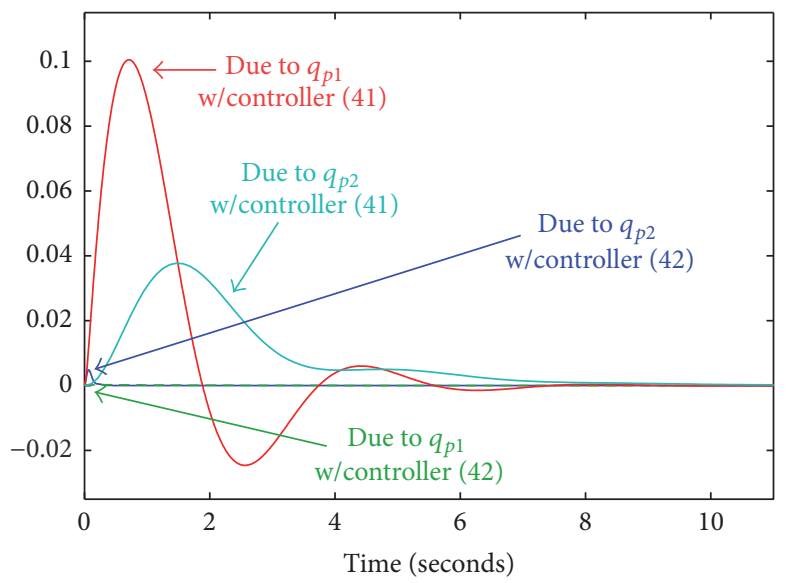

(a) $h_{1}(t)$

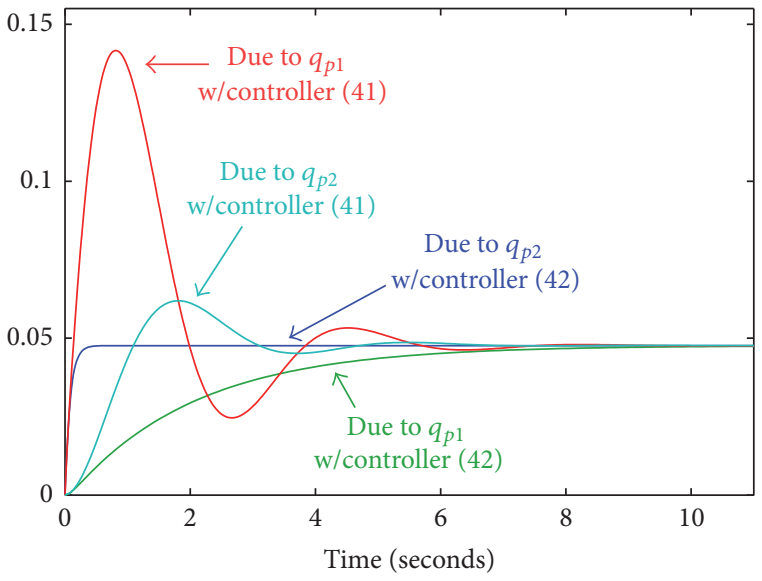

(b) $h_{2}(t)$

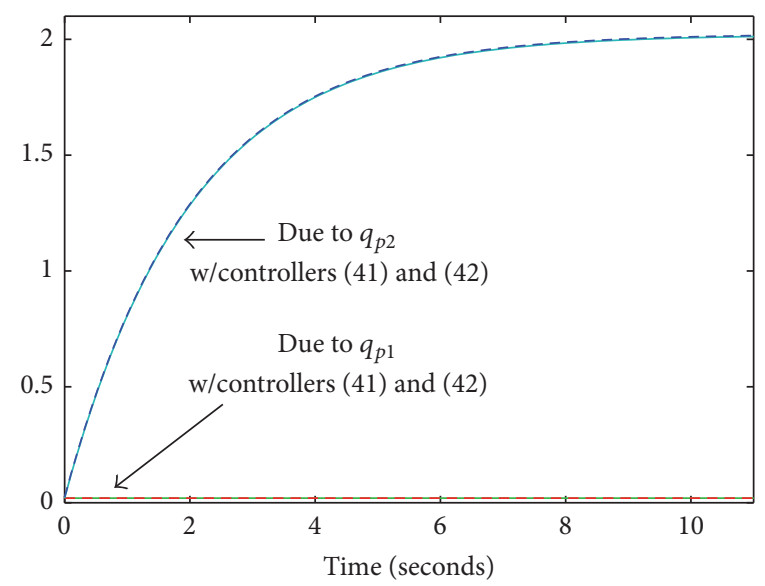

(c) $h_{3}(t)$

FIGURE 16: Full perturbation rejection assessment considering controllers (41) and (42).

\section{Acronyms}

SIMO: Single-Input-Multiple-Output

SISO: Single Input Single Output

MIMO: Multiple Input Multiple Output

ICAD: Individual Channel Analysis And Design
MSF: Multivariable Structure Function

TFM: Transfer function matrix

MPR: Main loop perturbation rejection

OSPR: Open-loop secondary perturbation rejection 
CSPR: Closed-loop secondary perturbation rejection

high-MPR: $h\left(j \omega_{0}\right) \rightarrow 1$

high-CSPR: $c_{j, p i}\left(j \omega_{0}\right) \rightarrow 0$.

\section{Conflicts of Interest}

The authors declare that there are no conflicts of interest regarding the publication of this paper.

\section{References}

[1] O. S. Ebrahim, M. F. Salem, P. K. Jain, and M. A. Badr, "Application of linear quadratic regulator theory to the stator field-oriented control of induction motors," IET Electric Power Applications, vol. 4, no. 8, pp. 637-646, 2010.

[2] A. S. Bazanella and R. Reginatto, "Robust tuning of the speed loop in indirect field oriented control of induction motors," Automatica, vol. 37, no. 11, pp. 1811-1818, 2001.

[3] F. W. Burcham, T. A. Maine, C. G. Fullerton, and E. A. Wells, "Preliminary flight results of a fly-by-throttle emergency flight control system on an f-15 airplane," NASA Technical Memorandum NASA TM-4503, 1993.

[4] F. W. Burcham Jr., T. A. Maine, J. J. Burken, and D. Pappas, "Development and flight test of an augmented thrust-only flight control system on an md-11 transport airplane," NASA Technical Memorandum NASA TM-4747, 1996.

[5] P. Lu and J. J. Burken, "Controlling aircraft with engine thrust only: nonlinear challenges," Nonlinear Analysis, vol. 35, no. 1, pp. 21-35, 1999.

[6] C. Spelta, F. Previdi, S. M. Savaresi et al., "Performance analysis of semi-active suspensions with control of variable damping and stiffness," Vehicle System Dynamics, vol. 49, no. 1-2, pp. 237256, 2011.

[7] G. Chen, J. Chen, and R. Middleton, "Optimal tracking performance for SIMO systems," IEEE Transactions on Automatic Control, vol. 47, no. 10, pp. 1770-1775, 2002.

[8] L. A. Amézquita-Brooks, J. Licéaga-Castro, E. Licéaga-Castro, and C. E. Ugalde-Loo, "Induction motor control: multivariable analysis and effective decentralized control of stator currents for high-performance applications," IEEE Transactions on Industrial Electronics, vol. 62, no. 11, pp. 6818-6832, 2015.

[9] D. T. McRuer and D. E. Johnston, "Flight control systems properties and problems, Volume I," NASA Contract Report NASA-CR-2500, 1975.

[10] D. E. Johnston, "Flight control systems properties and problems, Volume II block diagram compendium," NASA Contract Report NASA-CR-2501, 1975.

[11] C. Y. Xia, "Conjecture about the absolute stability problems of SIMO and MISO systems and a proof of its deduction," in Proceedings of the IEEE Control Theory and Applications, vol. 148, pp. 299-305.

[12] I. V. Kapalin and V. V. Fomichev, "Minimal stabilization of vector (MISO and SIMO) systems," Differential Equations, vol. 47, no. 11, pp. 1573-1582, 2011.

[13] T. Bakhtiar and S. Hara, " $\mathrm{H}_{2}$ regulation performance limitations for SIMO linear time-invariant feedback control systems," Automatica, vol. 44, no. 3, pp. 659-670, 2008.

[14] J. Freudenberg and R. Middleton, "Properties of single input, two output feedback systems," International Journal of Control, vol. 72, no. 16, pp. 1446-1465, 1999.
[15] L. Sun, J. Dong, D. Li, and K. Y. Lee, "A practical multivariable control approach based on inverted decoupling and decentralized active disturbance rejection control," Industrial and Engineering Chemistry Research, vol. 55, no. 7, pp. 2008-2019, 2016.

[16] L. Amezquita-Brooks, E. Liceaga-Castro, J. Liceaga-Castro, and C. E. Ugalde-Loo, "Flux-torque cross-coupling analysis of FOC schemes: novel perturbation rejection characteristics," ISA Transactions, vol. 58, pp. 446-461, 2015.

[17] J. O'Reilly and W. E. Leithead, "Multivariable control by individual channel design," International Journal of Control, vol. 54, no. 1, pp. 1-46, 1991.

[18] B. Yang, M. Wan, and Q. Sun, "Control of handling stability in four-wheel steering vehicles based on individual channel design," Key Engineering Materials, vol. 480-481, pp. 1074-1078, 2011.

[19] C. E. Ugalde-Loo and J. B. Ekanayake, "Individual channel analysis of induction generator-based wind turbines," in 2011 6th International Conference on Industrial and Information Systems, ICIIS 2011, pp. 277-282, lka, August 2011.

[20] E. Licéaga-Castro, J. Licéaga-Castro, C. E. Ugalde-Loo, and E. M. Navarro-López, "Efficient multivariable submarine depthcontrol system design," Ocean Engineering, vol. 35, no. 17-18, pp. 1747-1758, 2008.

[21] L. Amezquita-Brooks, E. Liceaga-Castro, and J. Liceaga-Castro, "The structural robustness of the induction motor stator currents subsystem," Asian Journal of Control, vol. 16, no. 6, pp. 1632-1645, 2014.

[22] J. C. Doyle, B. A. Francis, and A. R. Tannenbaum, Feedback Control Theory, Macmillan, New York, NY, USA, 1992.

[23] H. Kimura, "Robust stabilizability for a class of transfer functions," Institute of Electrical and Electronics Engineers. Transactions on Automatic Control, vol. 29, no. 9, pp. 788-793, 1984.

[24] J. S. Freudenberg and D. P. Looze, "Right half plane poles and zeros and design tradeoffs in feedback systems," IEEE Transactions on Automatic Control, vol. 30, no. 6, pp. 555-565, 1985.

[25] W. E. Leithead and J. O’Reilly, “Uncertain SISO systems with fixed stable minimum-phase controllers: relationship of closedloop systems to plant RHP poles and zeros," International Journal of Control, vol. 53, no. 4, pp. 771-798, 1991.

[26] L. Amezquita-Brooks, C. E. Ugalde-Loo, E. Liceaga-Castro, and J. Liceaga-Castro, "The multivariable structure function as an extension of the RGA matrix: relationship and advantages," Cybernetics and Physics, vol. 2, no. 2, pp. 53-62, 2013.

[27] W. E. Leithead and J. O'Reilly, "Investigation of the ICD structure of systems defined by state-space models," International Journal of Control, vol. 60, no. 1, pp. 71-89, 1994.

[28] O. Gasparyan, Linear and Nonlinear Multivariable Feedback Control: A Classical Approach, Wiley, Hoboken, NJ, USA, 2008.

[29] S. Skogestad and I. Postletwaite, Multivariable Feedback Control: Analysis and Design, Wiley, Hoboken, NJ, USA, 2nd edition, 2005. 


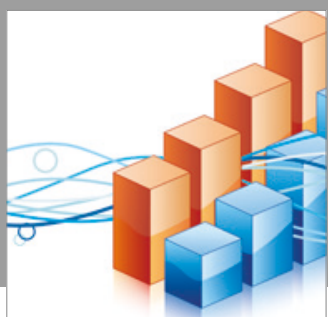

Advances in

Operations Research

vatersals

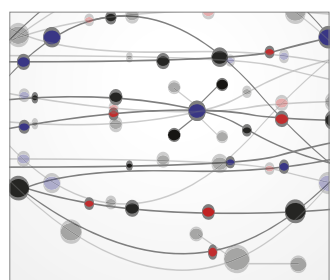

\section{The Scientific} World Journal
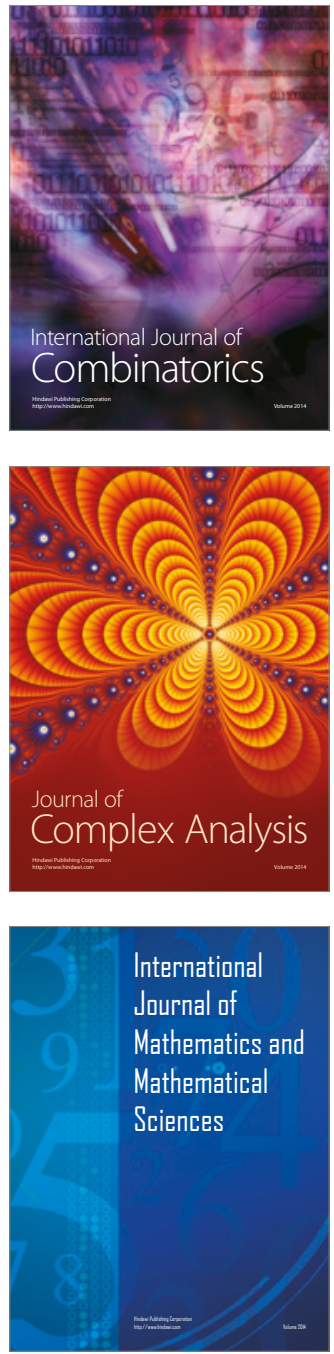
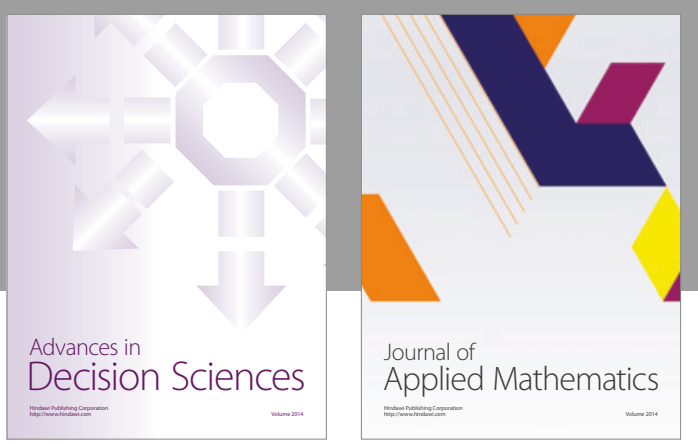

Algebra

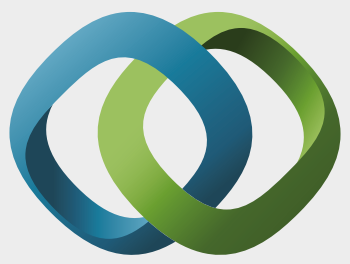

\section{Hindawi}

Submit your manuscripts at

https://www.hindawi.com
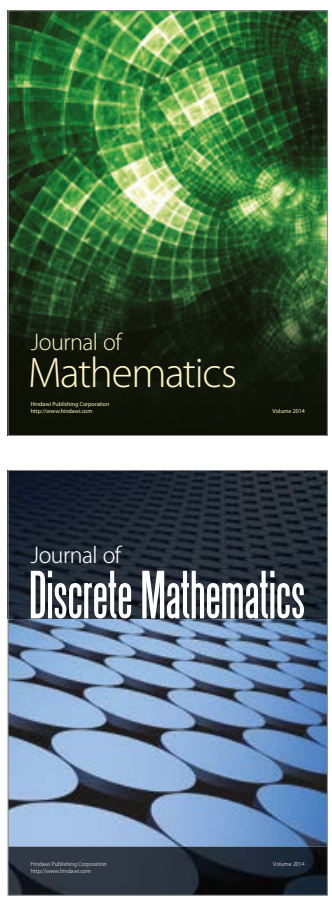

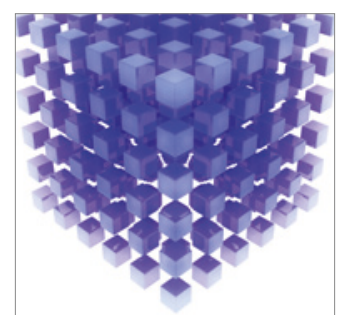

Mathematical Problems in Engineering
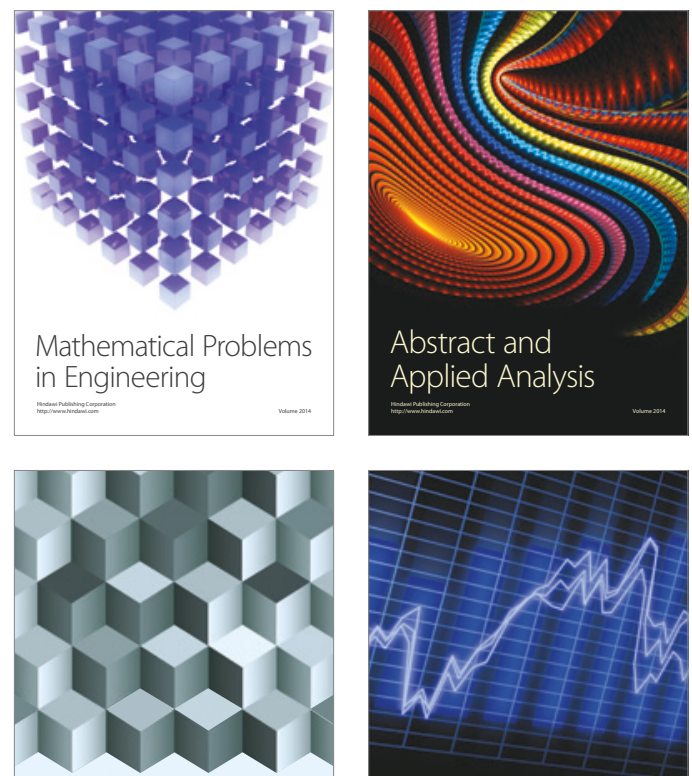

Journal of

Function Spaces

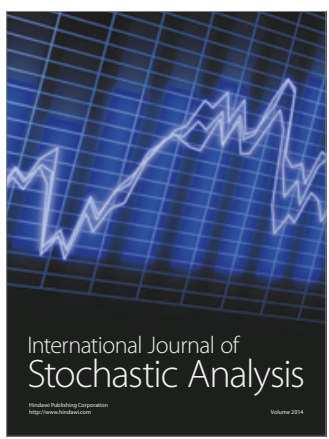

Probability and Statistics
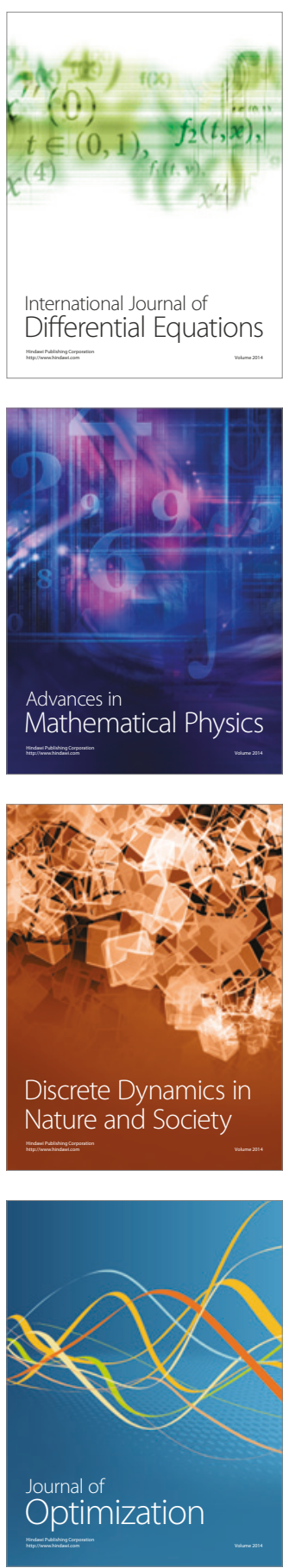